\title{
Modeling Languages
}

Modeling languages determine the concepts that can be used to describe an extract from perceived reality and how these concepts can be put into mutual relationship. Modeling languages thus provide the vocabulary and the grammar needed to represent real-life situations in models. They will subsequently be considered in a structured way.

The selection of a specific modeling language creates a well-defined point of reference for modeling, to which all actors involved - whether they are actively involved in the creation or passively affected by the model as consumers - can refer [1]. The term "actors" here is not necessarily restricted to humans, who are provided with a common, defined vocabulary by the modeling language in order to form a common understanding of the situation being modeled. It also refers to computer systems that are given the opportunity to further process models with automation support (e.g., in order to use them as a basis for workflow support), as the semantics of model elements are exactly specified in the modeling language (ibid.).

The choice of the modeling language therefore determines what can be represented in a model, and what cannot be made visible because the modeling language does not offer any appropriate concepts. The choice of modeling language also influences the usability of the model for different target groups [2,3]. Some modeling languages are designed for supporting communication among human actors and therefore may allow a "vague" representation of model semantics [4]. Other modeling languages require a more exact specification of semantics. They are preferably used to prepare models for use in IT systems. The choice of a modeling language therefore is dependent on the respective objective of the modeling process. It thus is a major step toward successful support of the activities the modeling process is embedded in.

In this chapter we provide the foundation for the appropriate selection of a suitable modeling language to fulfill the requirements of both human actors as well as machines and present different languages with their respective objectives and language elements. The focus here is placed on the mapping of business processes. All selected languages therefore allow the behavior of actors in 
organizations to be mapped in a broader sense. The languages, however, fundamentally differ in what is considered to be an "actor" and how the behavior of such can be described. The reasons for these differences can be found in the historical context of the languages and their respective objectives. The arrangement of the following sections therefore follows a historical perspective in order to clearly highlight the relationships between the languages and their origins. Finally, we discuss for each of the languages to which extent they allow the mapping of processes according to the definition used in this book, and where they have their respective focal points or gaps in representation capabilities.

\subsection{Overview}

Flowcharts, which are still used today, are one of the oldest modeling languages to describe processes. They were originally designed to represent the control flow in a computer program. Due to the generality of their language elements, they are also used for modeling processes in organizations and are therefore presented here as an introduction to graphical process modeling. They introduce the concept of branching in the control flow to represent alternative paths.

For a long time eEPCs ("extended Event-driven Process Chains") [5] were a de facto industry standard for Business Process Modeling in Europe. In addition to their capability to map process flows, they also include elements to include responsibilities, data or services in models. This enables the modeling of business processes in their organizational context. In addition, they allow explicit modeling of parallel activities and thus go beyond the expressiveness of flowcharts in the representation of the workflow.

Historically, Unified Modeling Language ( $U M L)$ activity diagrams can be seen as a further development of flowcharts to model software processes [6]. As part of the UML, they today represent the de facto standard for representing control flows in software. Like eEPCs, they also allow the mapping of parallel process flows. Using activity diagrams, we introduce the structuring of models through partitioning and nesting and show how models can be structured based on responsibilities and not just on the workflow itself.

$B P M N$ (Business Process Modeling Notation) is the predominant standard for representing business processes today [7]. Originally derived from several different modeling languages, including activity diagrams, BPMN was explicitly designed for representing business processes. In this context, the idea behind BPMN was that it should enable modeling with different objectives - from communication support to execution in Workflow Management Systems. From a conceptual language point of view, BPMN is particularly interesting with regard to its possibilities for compact representation of complex process flows (such as exception handling).

$S-B P M$ (Subject-oriented Business Process Modeling) is a modeling approach that puts the actors involved in a business process and their interactions at the center of the modeling process [8]. The resulting modeling language is characterized by a small number of language elements and comprehensive expressiveness for mapping 
business processes. On the one hand, it is presented here as an example of a not primarily flow-oriented approach to Business Process Modeling, and on the other hand, it represents, in terms of language conception, an alternative to BPMN with its very extensive set of modeling elements.

\subsection{Flowcharts}

Flowcharts allow the depiction of simple, sequential processes. A sequential process is characterized by the fact that no more than one activity is performed at any one time - thus parallel processes cannot be represented. Flowcharts were first described in the context of industrial production planning in the 1920s. At the end of the 1940s, they were adapted for the description of processes in the emerging Information Technology sector. Since the mid-1960s, they have been used as a standardized form of representation for computer program sequences. To this day, they are used to represent flows in computer programs or processes in organizations, as long as their complexity does not exceed the expressiveness of a flowchart.

The limitation of flowcharts' expressiveness is due to their historical development. In both industrial production planning and in early computer systems, it was not necessary to be able to represent parallel processes. Due to the limited computing resources available (only one CPU or only one processor core), there was no need to provide language elements to design parallel software processes.

\subsubsection{Notation Elements}

Flowcharts today exist in many different variants, which mainly differ in the notation used (i.e., the graphical representation). The semantics of the language elements, however, is common to all (cf., Figure 3.1). Basically, any number of operations (i.e., activities, represented as rectangles) is defined. These operations are put into an execution sequence by means of directed connections (represented as arrows). Rounded rectangles indicate the start and end of a process.

An essential means of describing processes, both in a computer program and in organizations, is the representation of alternative operations. The selection of an alternative usually depends on a condition that can be checked - in a computer program, this could be exceeding a limit for the value of a variable, in an organization, the existence of a particular document or the decision of a person responsible for executing the process could constitute the criterion for selecting an alternative. Alternatives are represented in flowcharts by branches (represented as diamonds),
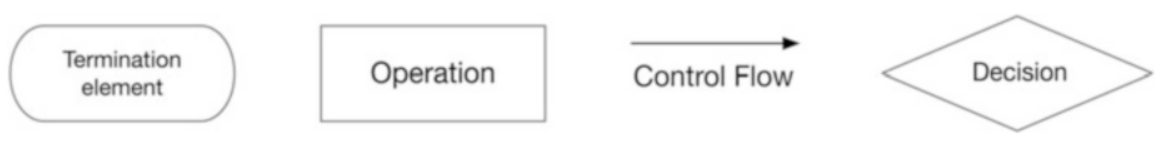

Figure 3.1: Notation of flowcharts 
which are connected to the previous operation via an incoming arrow and to the alternatively executed subsequent operations by two outgoing arrows. The restriction to exactly two (and not several) subsequent operations is also due to the origin of flowcharts from the representation of computer programs, since these usually are only capable of evaluating a condition in a binary way (i.e., as true or false). If a condition is to be checked for more than two values, several branches must be cascaded in flowcharts. The outgoing connections are labeled with the respective characteristic value at which the program is continued after the condition has been checked. A repeated execution of operations (for example, as long as there are still documents that need to be processed) is represented by jumping back to an earlier operation via an outgoing connection from a branch. The other outgoing connection then progresses to the operation that is executed when the repeated execution is complete.

In addition to these basic elements, flowcharts in some variants also offer special language elements that are used mainly to represent specific operations in the area of application (such as input or output operations in computer systems). However, these are not relevant for the conceptual understanding of flowcharts and especially not for their application in Business Process Modeling.

In the following, we present the use of the notation elements using examples from Business Process Modeling. The notation used is based on the symbols defined by ANSI and DIN in the 1960s.

\subsubsection{Examples}

The example in Figure 3.2 shows a process with a single operation in which an application (for which we have no detailed information here) is processed. The process ends after the processing of the application has been completed.

In the example in Figure 3.3, the process is extended by a decision. The application is checked, and the result of this check allows a decision to be taken on its confirmation or rejection. The confirmation or rejection itself are, in turn, operations. The outgoing connections are merged after the alternative branches are completed.

Figure 3.2: Simple

flowchart

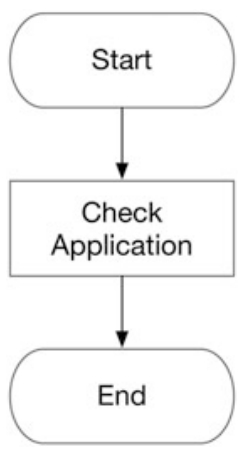


Figure 3.3: Flowchart with binary decision

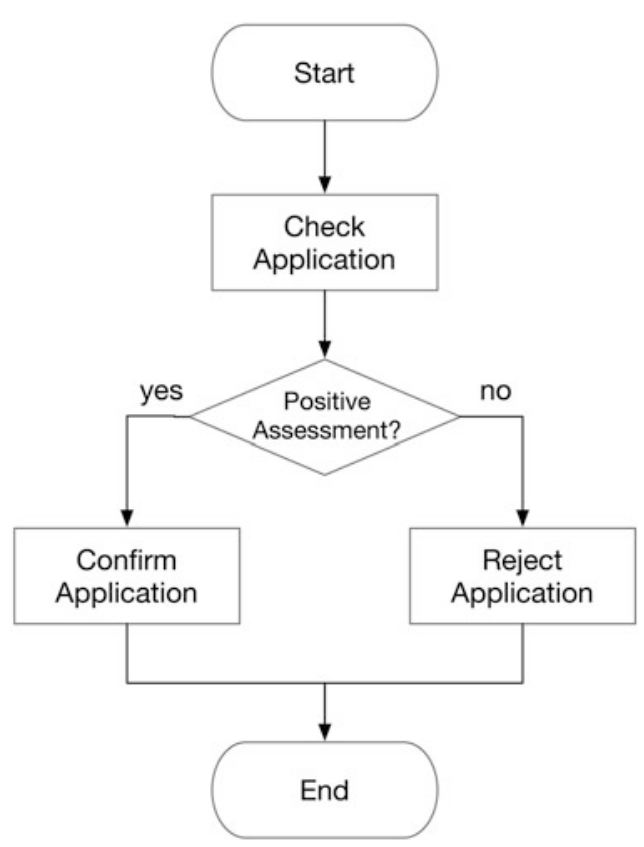

Decisions with more than two possible outputs must be represented in flowcharts using cascaded decision elements. This can be seen in the example in Figure 3.4. Obviously, our applications are investment requests. The first decision now examines whether the sum of investment costs is less than EUR 1,000. If this is the case, the request is confirmed directly. If this is not the case, a second decision is taken. It checks whether the application sum is less than EUR 10,000. If this is the case, the application sum is between EUR 1,000 and EUR 9,999, which leads to an examination of the attachments to the application. If the application amount is not less than EUR 10,000.-, i.e., EUR 10,000.- or more, the application will be forwarded. We don't learn anything about the destination of the forwarding here, because flowcharts don't offer notation elements for depicting this information.

As already mentioned, branches can also be used to repeat parts of a process (cf., Figure 3.5). To do this, the decision is inserted at the end of the part to be repeated and an outgoing branch leads back to the point prior to the first operation to be repeated. The other outgoing branch continues the process after the repetition is completed. In the above example, applications are processed as long as there are more applications available. It is important to understand that at least one application must be processed before a decision can be reviewed. If the process should be able to finish if there are no applications at all, an additional decision would have to be inserted at the beginning of the process ("Applications present?"), from which an outgoing connection ("no") leads directly to the end of the process. The other outgoing connection ("yes") would lead to the process flow previously described. 


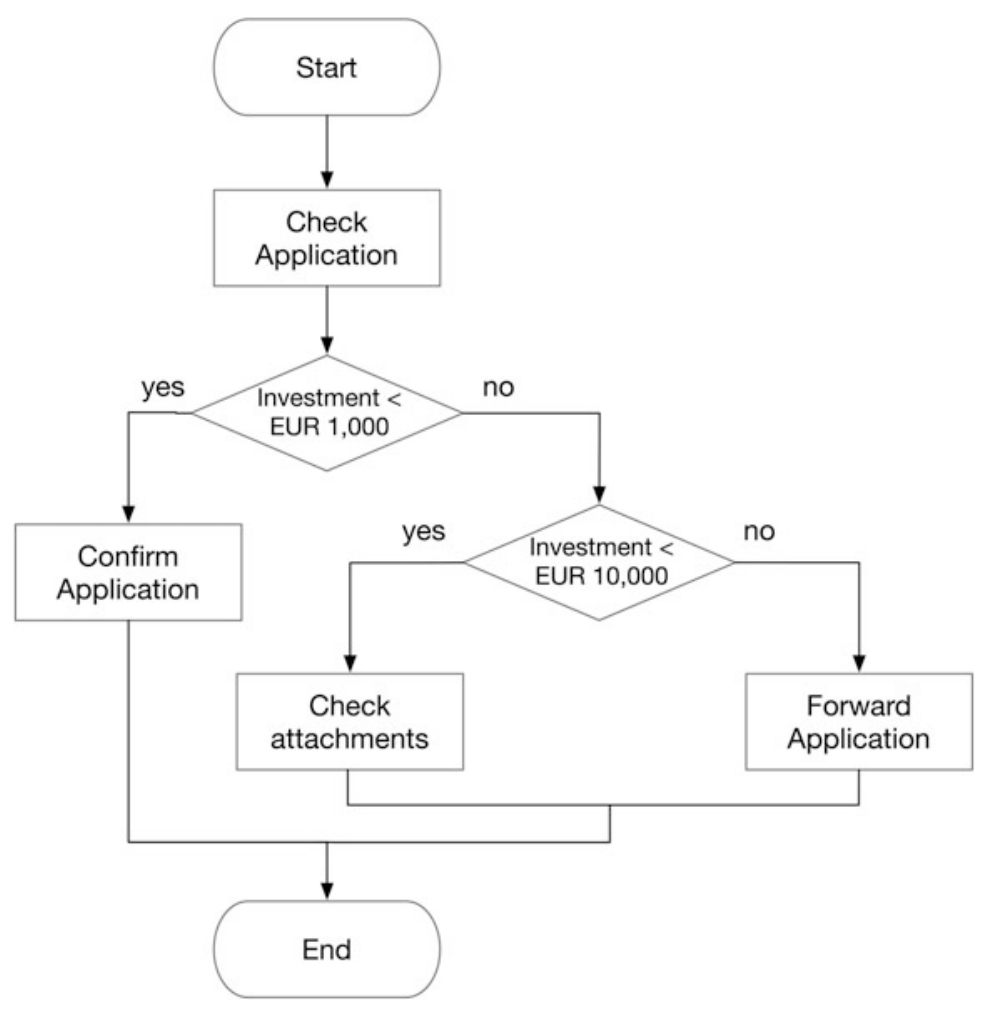

Figure 3.4: Flowchart with multiple-outcome decision

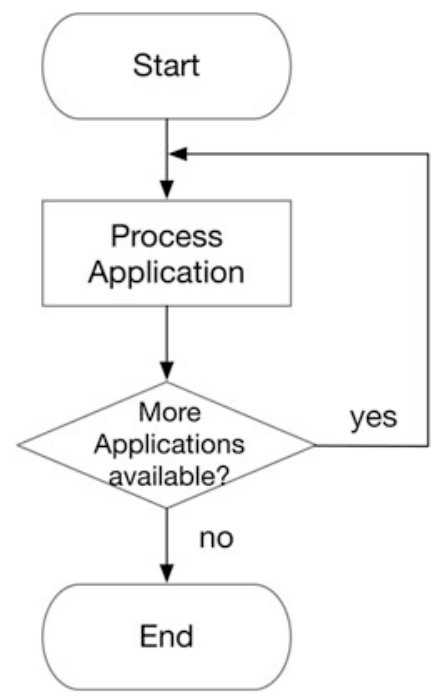

Figure 3.5: Flowchart with repeated execution of process parts 


\subsubsection{Classification}

Flowcharts are a simple way to represent business processes in terms of the logical sequence of activities they contain. Other aspects of a business process, such as data or responsibilities, are not accounted for in the language and cannot therefore be represented.

It is also not possible to represent parallel process flows. This is a major reason why flowcharts are partly being replaced by more recent languages such as UML activity diagrams or BPMN, which offer constructs for executing operations in parallel. Since flowcharts do not offer any element to depict responsibilities, it is not possible to represent communication processes - this restriction also is addressed in more modern languages, which we introduce in the following sections.

\subsection{Event-Driven Process Chains}

For a long time Event-driven Process Chains (EPCs) were the de-facto industry standard in Europe for representing business process models. They were developed as part of the ARIS concept already presented in Section 1.5 and there serve as a means of representing models of the control view of an organization - i.e., the view that deals with the processes in an organization and the associated links between its resources. Resources here can be both acting members and/or departments of an organization from an active perspective, as well as the required and/or manipulated data or goods from a passive perspective.

EPCs links the functions that an organization is capable of performing based on upcoming events during business operation. The basic principle of representation is that a function is always triggered by an event - that is, a function must always be preceded by an event in an EPC model in order to determine whether the execution of a function can be started. In the more comprehensive variant "extended EPC" (eEPC), the functions are linked to the elements of the other ARIS views relevant for their execution. In particular, the responsible actors, roles, or organizational units can be assigned from the organizational view, and the relevant documents or data objects from the data view. If a function leads to a billable service, this can be represented by elements taken from the service view.

In addition to the representation of decisions, EPCs also enable the mapping of parallel process flows of a business process. For this purpose, it provides additional notation elements. By way of AND connectors process branches run in parallel can be displayed. The XOR connector is used to represent decisions for which exactly one alternative has to be selected (this corresponds to the decision element in flowcharts). The OR connector can be used to represent processes in which one or more alternatives can be chosen. 


\subsubsection{Notation Elements of EPCs}

The basic element for describing business processes in EPCs is the function (similar to the operation in flowcharts, cf., Figure 3.6). However, the sequence of functions in a process is not determined exclusively by connection arrows. By using events the sequence is specified here in more detail. Every function is triggered by an event and subsequently creates one or more events itself. A process is thus represented by a sequence of events and functions, whereby events and functions always alternate. When naming these, functions should always describe a performable activity (e.g., "Check request"), while events should always describe a state (e.g., "Confirmed request" or "Rejected request").

An EPC always starts and ends with an event. Events that trigger a process are referred to as start events. Events that describe the completion of a process are referred to as end events. Subsequent processes can be triggered by end events of a previous process, that is, an end event can be a triggering start event in another process.

By using different connectors in combination with events, it is possible to represent different control flow variants of a process within a model. This includes the possibility of executing functions in parallel (if these are not dependent on each other). The AND, OR and XOR connectors can be used for this purpose:

If an AND connector with several outgoing connections is used, all outgoing paths are traversed in parallel. These paths are then usually joined at a later point in time with another AND connector. The function after the joining AND connector is not executed until all the incoming paths have been completed.

An OR connector with multiple outgoing connections indicates that one or more of the following paths are traversed in parallel. These paths are usually joined again at a later point with another OR connector, whereby the subsequent function is only carried out when exactly those paths chosen at the original OR branch have been completed. It is important here that the paths to be activated must be selected at the time of reaching the OR connector - it cannot be used to trigger further paths at a later point in time, should this become necessary during execution. Each OR connector must be followed by an event in each of the paths that could be triggered by the function preceding the OR connector. The paths whose first events actually occur are activated during runtime.

Finally, an XOR connector is used for representing "exclusive OR" or "either, or" decisions. Using an XOR connector with multiple outgoing connections means that exactly one of the following paths is selected when the process is executed. It is therefore suitable for representing mutually exclusive alternatives in processes. The

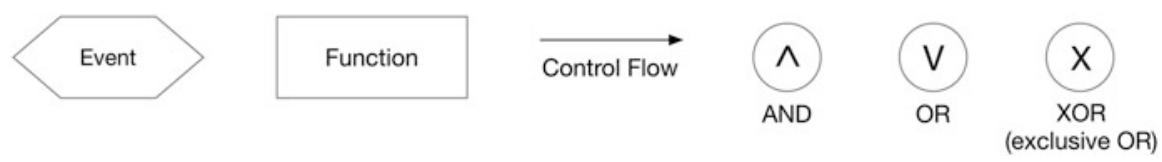

Figure 3.6: Notation of EPCs 
paths are joined again with an XOR connector, and the subsequent function is performed when the selected path has been completed. As for OR connectors, XORs must also be followed by an event on each path that could be triggered by the preceding function. These events must be mutually exclusive. The path with the first event that actually occurs is activated during runtime. In contrast to flowcharts, it is also possible to describe more than two alternatives here, as long as the events used are mutually exclusive.

\subsubsection{Examples of EPCs}

We here use the same examples as shown for flowcharts to visualize the differences in the notation.

The example in Figure 3.7 shows a process with a single function in which an application (for which we have no further information here) is processed. The process ends after the application has been checked.

In the example in Figure 3.8, the process is extended by a decision. The application is checked, and the result of this check allows making a decision on its positive or negative assessment. The confirmation or rejection itself are, in turn, functions. The outgoing connections are joined with an XOR connector after the alternate branches are completed.

The representation of decisions that have more than two possible options is easier here than with flowcharts. In Figure 3.9, the XOR connector is followed by three events that all refer to the investment amount and are mutually exclusive.

The XOR connector can also be used to repeat parts of a process (cf., Figure 3.10). To do this, the connector is inserted at the end of the part to be repeated and an outgoing connection (line) links back to the event that triggers the part to be repeated. The other outgoing branch continues the part of the process to be executed after the repetition has been completed. It must lead to an event that triggers the termination of the repetition. In the above example, applications are processed as long as further applications are available.

Figure 3.7: Simple EPC

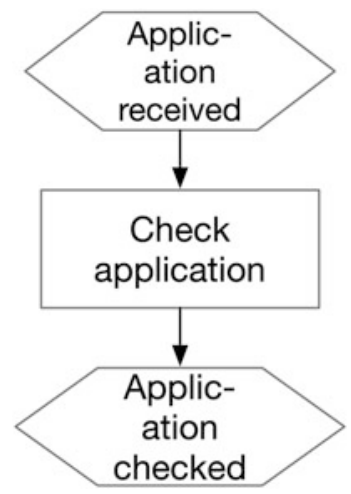


Figure 3.8: $\mathrm{EPC}$ with binary decision

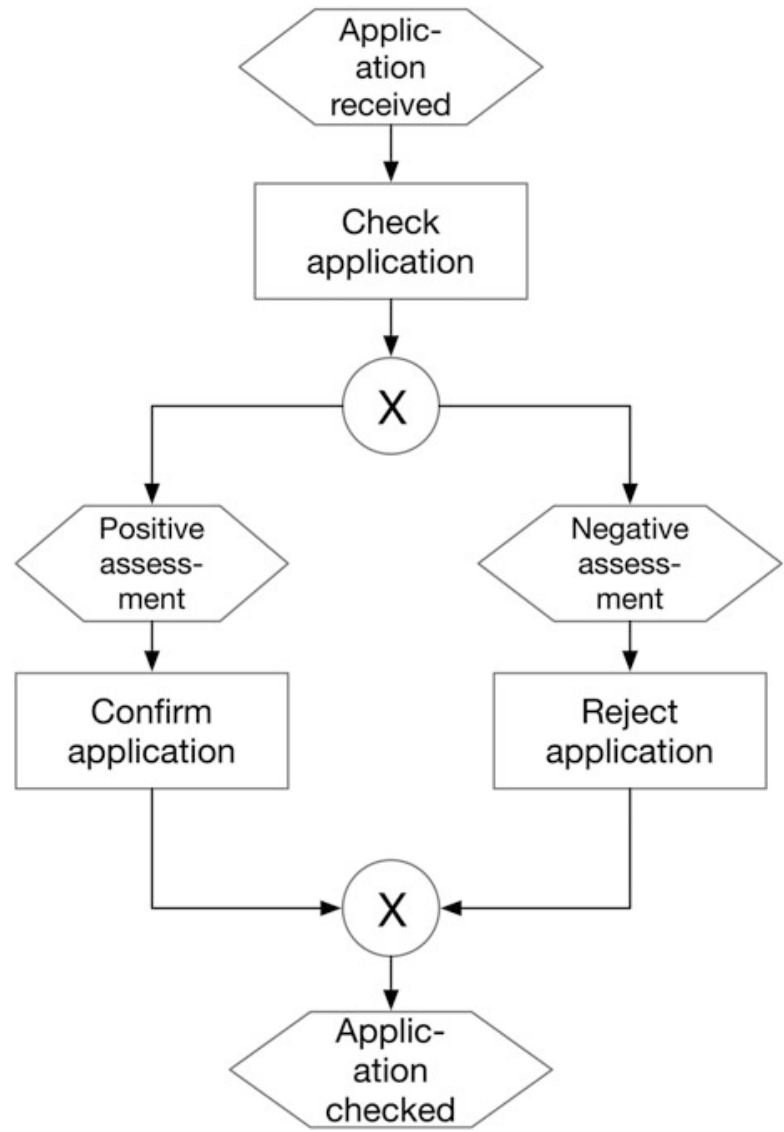

The AND connector can be used if two functions can be executed independently of each other (cf., Figure 3.11). The process modeled in the above example is therefore only correct if the attachments can actually be checked independently of the application. If this is not the case, the two functions would have to be arranged sequentially. From the point of view of modeling, the AND connector, unlike the other connectors, does not have to be immediately followed by events, since no decision is made. All outgoing branches are activated in any case.

Figure 3.12 shows an example of the use of the OR connector. Here we assume that an application may contain offers or comments, or both (but must at least contain offers or comments). If offers and comments were completely optional (i.e., if both could be missing), their fundamental necessity would have to be examined with an upstream XOR connector and corresponding events. Alternatively, an additional branch could be added to the OR connector with an event "Application alone is sufficient". In both cases, the condition that events and functions must alternate on all paths through the process must not be violated. If not otherwise possible, this must be ensured by a "dummy" function that does not lead to any actual activity. 


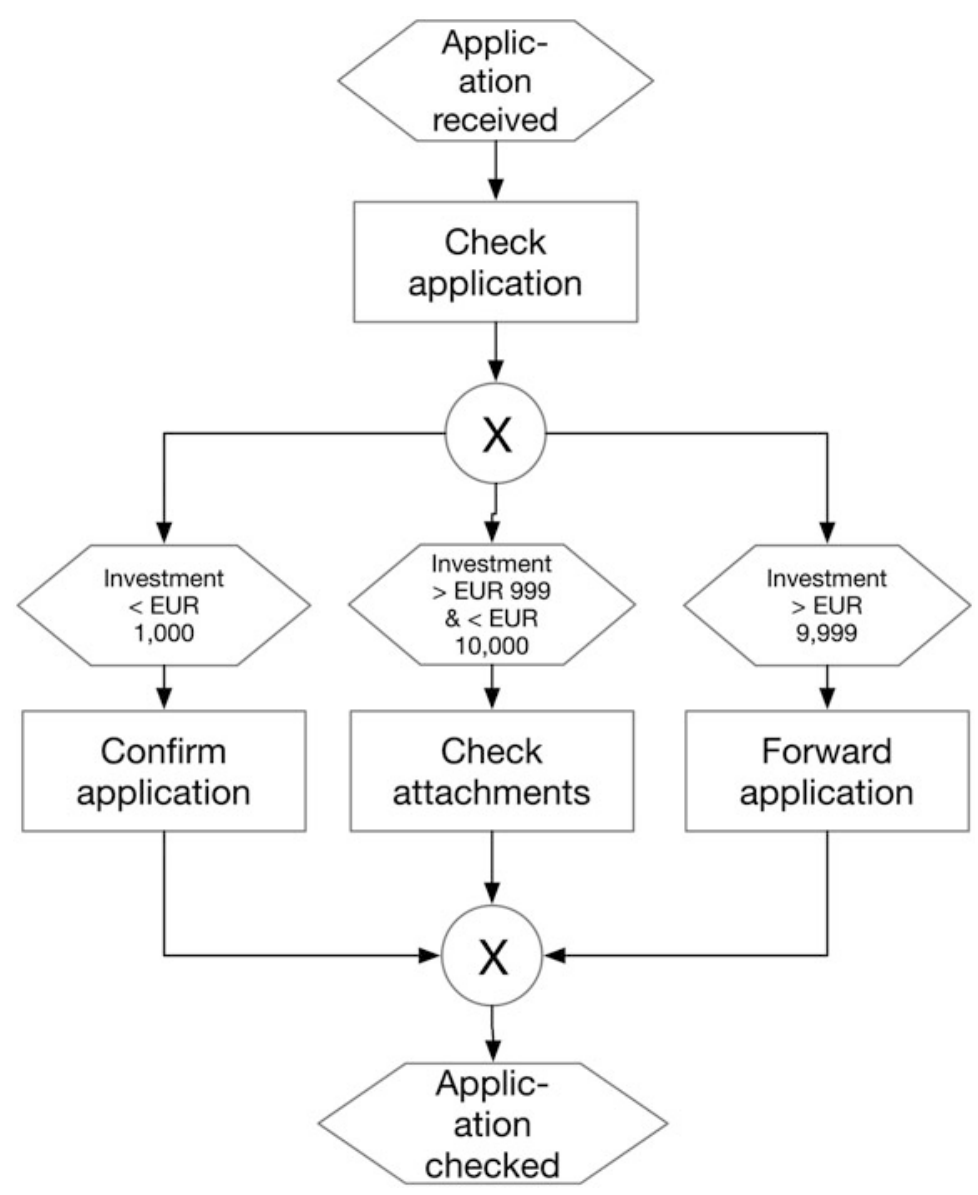

Figure 3.9: EPC with multi-outcome decision

\subsubsection{Supplementary Notation Elements in eEPCs}

The eEPC supplements the business process depicted in an EPC with information about its execution context. In particular, responsibilities and resource requirements are assigned to the functions here. The basic rules of an EPC remain unchanged. The additional elements can only be assigned to functions - events are not affected. At this point we do not provide a complete description of the possible elements of EPCs but focus on the most common ones (cf., Figure 3.13).

Responsibilities are represented by organizational units. Such units do not usually represent concrete persons but abstractly identify the name of a role (e.g., managing director) or department (e.g., financial accounting). This ensures that the specification of a process is independent of the availability of concrete staff resources. Concrete persons do not have to be assigned until the time of execution. 
Figure 3.10: $\mathrm{EPC}$ with repeated execution of process parts

Figure 3.11: EPC with parallel execution of process parts
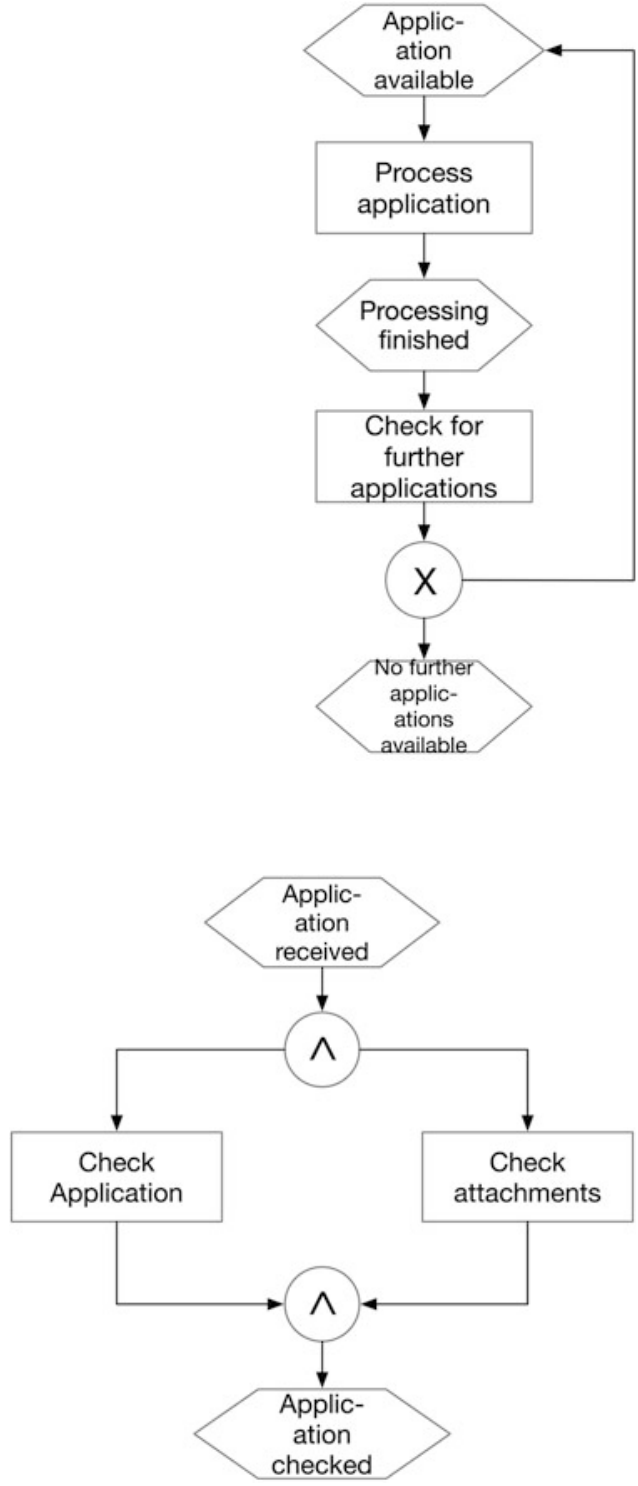

Undirected connections (lines) are used to show the assignment to a function. In this way, an organizational unit can be assigned to several functions. It is also possible to list organizational units more than once, if the model layout can be made clearer in this way.

IT systems are modeled in a similar way. They indicate the need to use a particular IT system (for example, an ERP system or a database) when executing a function. They are also assigned to functions with undirected connections (lines). 


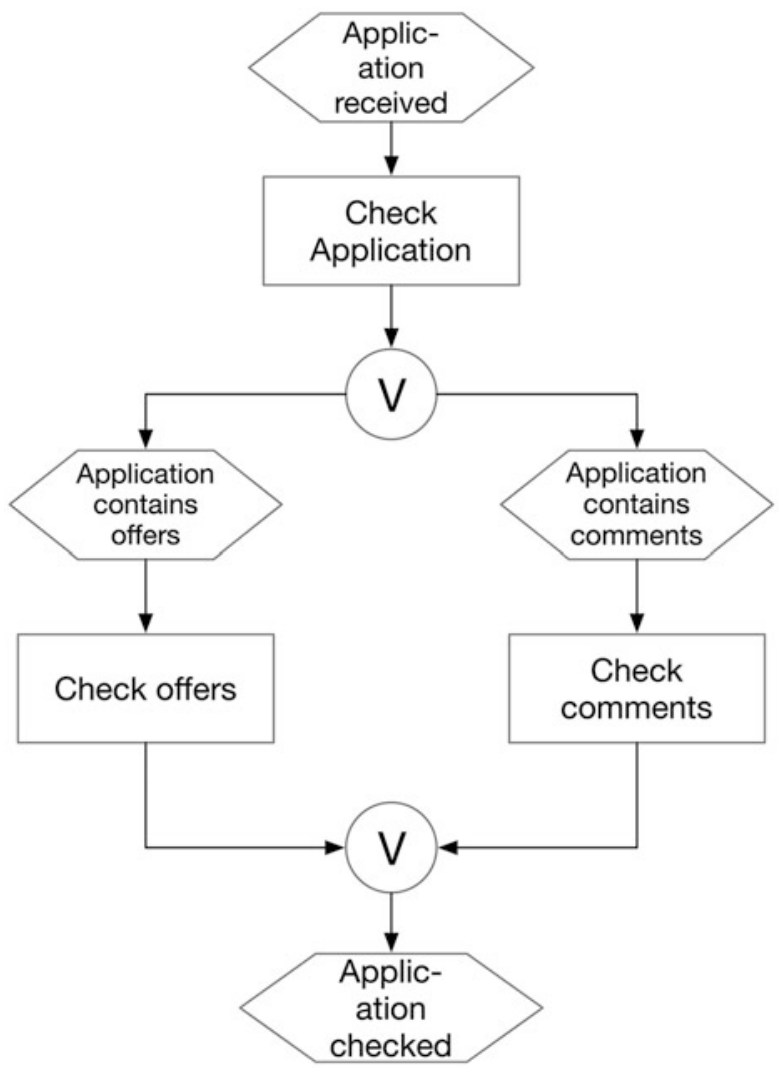

Figure 3.12: EPC with optional parallel execution of process parts

Org. unit
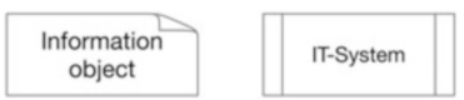

Figure 3.13: Additional notation elements for eEPCs

Information objects are used to represent data processing in a business process. An information object can be arbitrarily comprehensive (i.e., a single value, as well as a complete document) and is always assigned to a function by means of a directional connection (arrow) that describes the data flow. If the arrow ends at the function, this means that the information object is required to execute the function. If the arrow ends at the information object, this means that it is created or changed by the function. An information object can have several inbound and outbound connections, which can describe both its origin and its use in a business process. 


\subsubsection{Example of an eEPC}

To illustrate eEPCs, we use one of the examples described above and add the data flow and the human resources required (cf., Figure 3.14). Application systems would be modeled analogously to the use in organizational units.

With regard to the data flow, we can now see that the actual application must be present in order to check the application. This check not only leads to a positive or negative assessment of the application in the process flow, but also to an information object in which the assessment is stored. In the case of a negative assessment, this information object is required to create the rejection (we can therefore assume that the rejection contains a substantive reason). If the application is confirmed, the "Assessment" data object is no longer required - so we can assume that no further justification will be given in this case.

With regard to responsibilities, we now recognize that several organizational units are involved in the process. While the application is checked by a clerk, the head of department is responsible for the final confirmation or rejection. It is important here that the events on which the decision is based are triggered by the "Check application" function, for which the clerk is responsible. In the present process model, the head of department has no possibility to revise the decision once made.

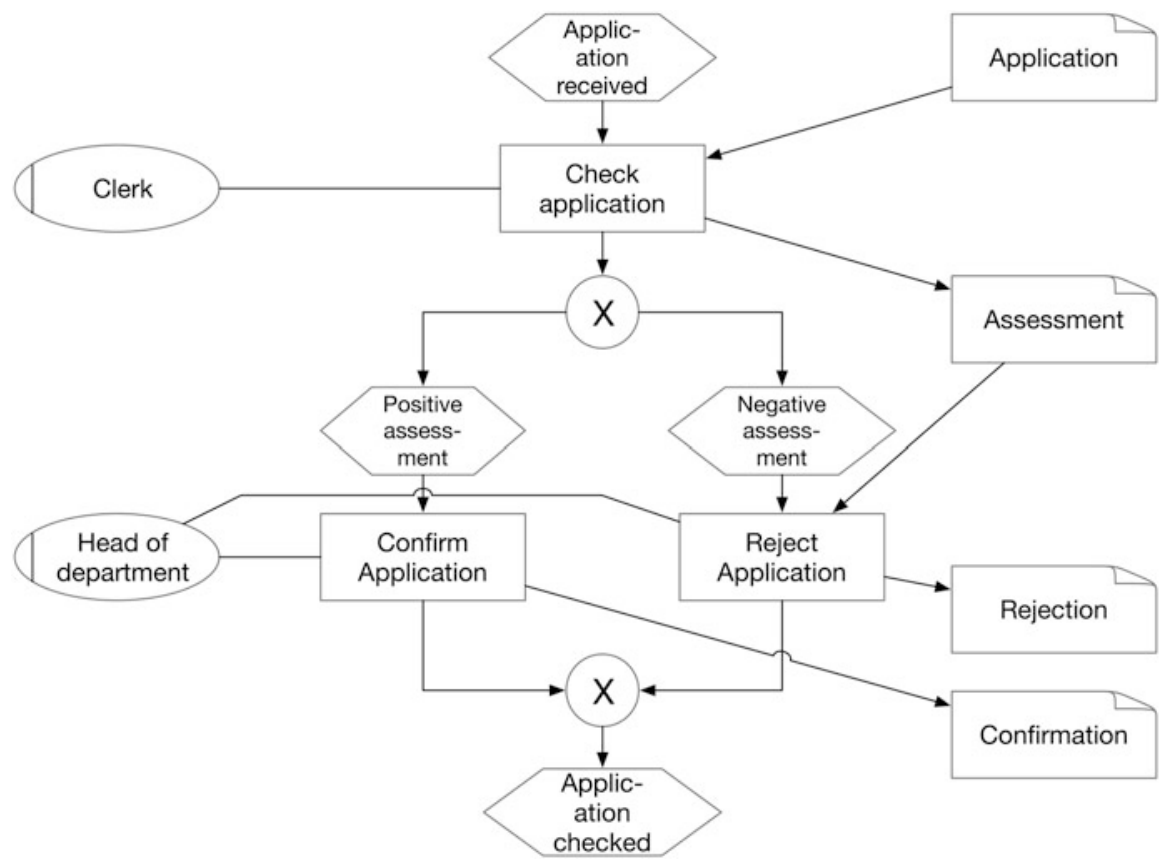

Figure 3.14: Example of an eEPC 


\subsubsection{Discussion}

The EPC offers more comprehensive possibilities for representing business processes than the flowchart. What they have in common is their orientation toward using the tasks and activities within an organization as the primary structuring characteristic of the business process (i.e., all information depicted in the model is anchored in the description of the process flow). This is an obvious choice when describing organizational processes, but - as we will see later - not necessarily the only possibility. Other modeling languages use actors or data as their primary structuring elements on which all other information is anchored. This makes aspects of the process visible that can only be implicitly represented in (e)EPCs (such as the transition between responsibilities in the process and the necessary communication between the actors).

The requirement to alternate functions and events in the process flow in EPCs often leads to very extensive models that are sometimes difficult to understand. It also bears the risk of tempting modelers to formulate trivial events that do not add any information to the model (e.g., function: "Execute task", event: "Task executed"). When used correctly, however, the EPC approach offers advantages: On the one hand, processes can be described and delimited more precisely than with flowcharts; on the other hand, EPCs explicitly allow the view on the capabilities of an organization (its functions) to be linked to the view on how it uses these capabilities to react to external stimuli or events within the organization itself. Thus, organizational capabilities can be described generically and used multiple times in processes, avoiding inefficiency through replication. From a pragmatic point of view, however, practical experience has shown that the specification of generic functions, as well as process-specific events, in the necessary detail is not always feasible. More modern approaches, such as the activity diagrams discussed in the following or BPMN, therefore still use the concept of events, but only deploy these if an external stimulus (such as an incoming message, an error, or a deadline) actually needs to be addressed.

In contrast to the modeling languages with a technically oriented history (such as flowcharts or the activity diagrams described in the next section), the eEPC and the surrounding ARIS framework are concepts originally derived from business administration. They thus pursue a more comprehensive approach to the description of business processes than the technology-centric approaches. The consideration of data, responsibilities, but also goals or services (which were not discussed here), enables a comprehensive modeling of business processes, which still influences the design of contemporary modeling languages for the representation of organizational phenomena (such as business processes or enterprise architectures). 


\subsection{UML Activity Diagrams}

The activity diagram is defined as part of UML (Unified Modeling Language), which contains a collection of modeling diagrams suitable for specifying software systems. The activity diagram there takes a role similar to that of the original flowchart and is used to illustrate the behavior of a software system. Because of its more recent historical context, it also provides elements for the illustration of distributed and parallel process flows. Like the flowchart, the activity diagram is also suitable for representing organizational processes (i.e., business processes). While it is still used for this purpose today, the focus in the area of Business Process Modeling has shifted strongly toward BPMN (Business Process Modeling Notation). BPMN was specified by the same standardization body as UML and has adopted many elements of the activity diagram. However, BPMN focuses more explicitly on the requirements of Business Process Modeling and the organizational aspects to be represented there, which we have already discussed for EPCs.

\subsubsection{Notation Elements}

By definition, an activity diagram always describes an activity that consists of individual actions ("activity" is thus used here analogously to "process"). An action corresponds to an operation for flowcharts, or a function for EPCs, cf., Figure 3.15).

An activity usually begins with a start node and finishes with an end node (similar to the associated elements for flowcharts). Between these nodes, the actions contained are specified and brought into sequence by control flow arrows. To influence the process, it is possible to insert decision elements. Decisions can have any number of outgoing branches, for which the activation conditions must be mutually exclusive. The conditions are listed at the outgoing connections. The semantics of the decision symbol corresponds to the XOR in the EPC - there is no equivalent for the OR connector in activity diagrams.

The activity diagram provides the split/join element to represent process parts that can be executed independently of each other and in parallel. When used to split the process, it can have any number of outgoing connections that are all activated at the same time. The branches created in this way should be merged again by the join element. The process is not continued until all branches have been finished.

Figure 3.15: Notation elements of activity diagrams

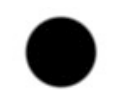

Start Node

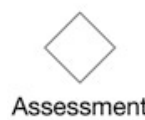

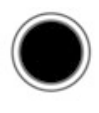

End Node

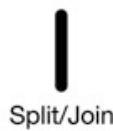

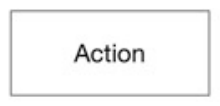

Control Flow

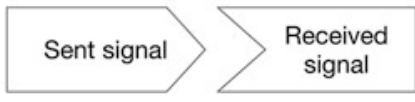


Figure 3.16: Notation elements to represent responsibilities and data flows in activity diagrams

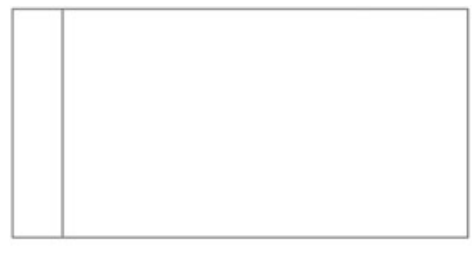

Data object

Partition

Signals are used for communication between process parts in different activities (i.e., in different diagrams), or within an activity when information is to be provided for subsequent process parts. They are incorporated into the control flow like actions. Models do not always have to contain complete signal pairs (i.e., send and receive signals). They can also send signals for processes that are not shown in the diagram (i.e., contain only a send signal) or receive signals from a process that is not shown in the diagram (i.e., contain only a receive signal). Received signals can also trigger an activity and thus replace the start node in a diagram.

The activity diagram also provides elements to represent responsibilities and data flows (cf., Figure 3.16). Responsibilities are represented using partitions. Partitions are elements that enclose parts of an activity diagram and thus determine that all elements, in particular the actions they enclose, fall under the responsibility of the stated organizational unit (or, in the case of software systems, system component). If partitions are used (which is not mandatory), all elements of the activity diagram should be enclosed by exactly one of the partitions shown. Overlaps are not allowed, actions outside a partition should be avoided.

Data objects in activity diagrams are directly incorporated into the control flow between actions. They can therefore (only) be used to represent the flow of information between two consecutive actions. If a data object is only needed again later in the process flow, it has to be forwarded in the control flow via all intermediate actions, or passed on by means of a signal.

\subsubsection{Examples}

In order to work out the differences and similarities to the previously discussed modeling languages, we again make use of the examples already used above.

The example in Figure 3.17 shows an activity with a single action in which an application (for which we have no further information here) is processed. The activity ends after the processing of the application has been completed.

In the example in Figure 3.18, the process is extended by a decision with three possible outcomes that are mutually exclusive. The application is checked, and the result of this check allows the decision to be taken with regards to the further processing of the application.

As with the other modeling languages, we can also use branches here to repeat parts of a process (cf., Figure 3.19). For this purpose, the decision is inserted at the 
Figure 3.17: Simple acitivity diagram

Figure 3.18: Activity diagram with a multipleoutcome decision

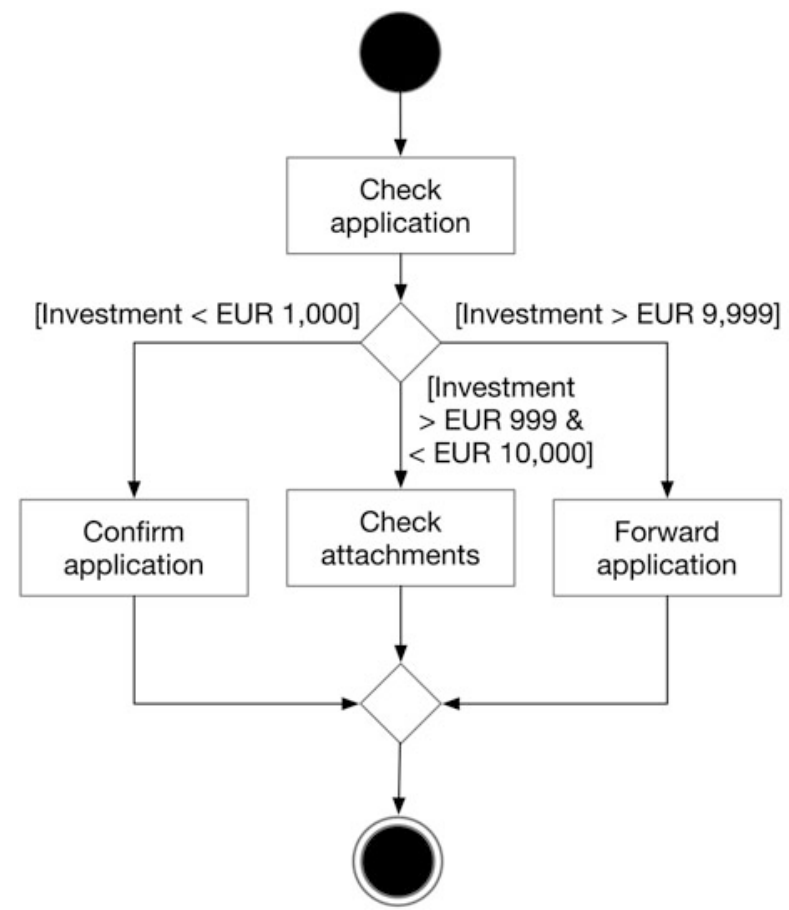

end of the part to be repeated and an outgoing branch is linked back to a closing decision before the first operation of the part to be repeated (a closing decision is used for joining alternative branches and has several incoming and only one outgoing connection). The other outgoing branch of the opening decision element continues the process after the repetition is completed.

The split/join element can be used if two action sequences can be executed independently of each other (cf., Figure 3.20). The process modeled in the above example is therefore only correct if the attachments can actually be checked independently of the application. If this is not the case, the two actions would have to be 
Figure 3.19: Activity diagram with repeated execution of process parts (loop)

Figure 3.20: Activity diagram with parallel

execution of process parts
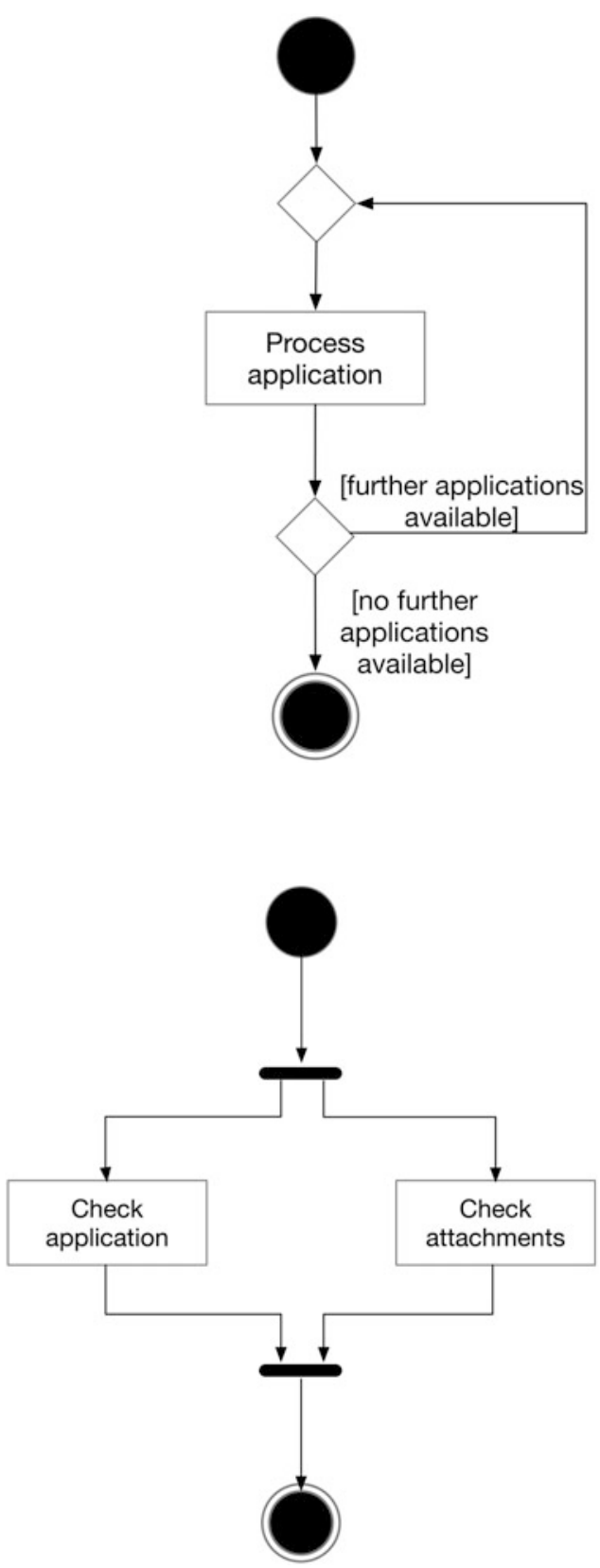

arranged sequentially. The merging join element waits for both incoming branches to be completed before continuing the process.

The example in Figure 3.21 demonstrates the use of partitions, data objects and signals. Partitions are used to represent the responsibilities in the process. The process is triggered by a received signal, which explicitly shows that the execution 


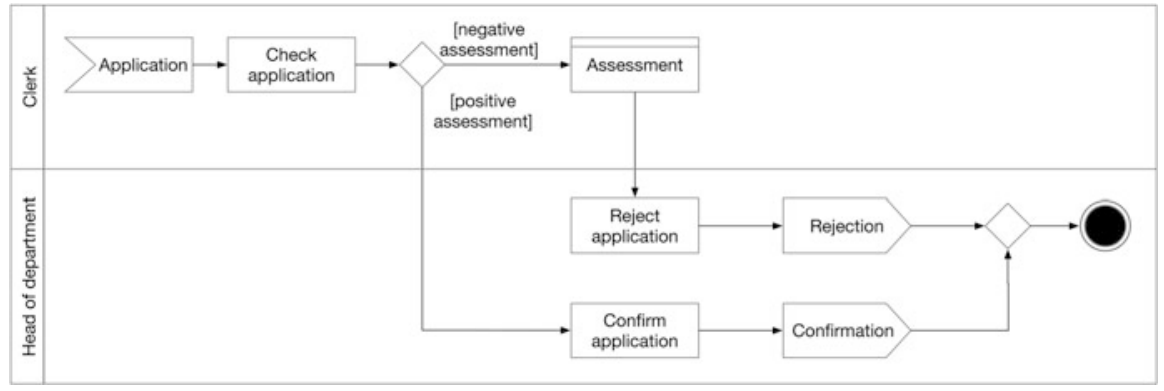

Figure 3.21: Example of an acitivity diagram with partitions, data objects and signals

only starts when an application is received (we had previously always implicitly assumed this for the flowchart and the other examples given for the activity diagram, but, unlike EPCs, were never able to represent it in the model). Send signals are used to transmit the confirmation or rejection to the recipient (who is not shown here). The assessment of the application is only transferred in the case of a negative assessment as a data object for the action "Reject application" (we can therefore assume that the rejection contains a substantive reason). If the application is confirmed, the "Assessment" data object is not required, so we can assume that no further justification will be given in this case.

\subsubsection{Classification}

Activity diagrams largely combine the simplicity of flowchart notation with the expressiveness of EPCs (with certain limitations). They allow the handling of data in the process to be represented and introduce a means of clearly representing responsibilities with the partitions. In contrast to the previously discussed languages, the availability of signals allows the depiction of communication processes between participants or with the surrounding environment of the displayed process.

The absence of an element corresponding to the OR connector in the EPC does indeed represent a limitation, however this is rarely relevant in practice, since we are usually confronted with mutually exclusive alternatives or completely independent branches of execution in the real world. Activity diagrams are therefore a suitable tool for representing business processes on the whole, especially if the target group using the model has an Information Technology background and is already familiar with the notation. For other target groups, BPMN, which we will discuss in the next section, is preferable due to its more flexible applicability and higher expressiveness for Business Process Modeling. 


\subsection{BPMN}

BPMN - the modeling language referred to as Business Process Modeling (and) Notation - was developed by IBM in 2002 and subsequently published by the BPMI (Business Process Management Initiative). The aim was to create a universally applicable standard to counter the multitude of process modeling languages used in academia and industry. This language should adopt the essential characteristics of the most common languages and make it possible, in addition to the documentation of business processes, to create models that allow for immediate IT-supported execution. BPMI in turn was merged with the OMG (Object Management Group) in 2005. Thus, BPMN became an OMG standard and complements the already mentioned UML (Unified Modeling Language), which is maintained by the same group.

The BPMN 2.0 standard was published in 2010. This standard incorporates several new diagram types: the choreography diagram, the conversation diagram, and the collaboration diagram. In the following, we consider the basic elements of BPMN 2.0 that enable business processes to be represented at the business level.

BPMN focusses on business processes, which it presents as a temporally logical sequence of activities (tasks) which are structured in accordance with organizational responsibilities. The representation of data is not as comprehensive as in other modeling languages and only regarded in the context of process flows.

\subsubsection{Notation Elements for Modeling Process Flows}

Process diagrams created with BPMN are called Business Process Diagrams (BPD). In its core BPD follows the principles of activity diagrams, which are subsequently supplemented by elements that allow the representation of the potentially more complex control flow in business processes (cf., Figure 3.22).

As a basic principle of representation, certain things have to be done in a process (tasks), but possibly only under certain conditions (gateways), and things can happen

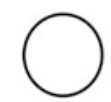

Start event

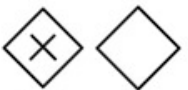

Exclusive gateway

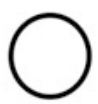

End event

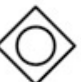

Inclusive gateway

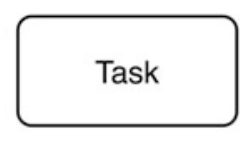

$\longrightarrow$

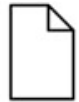

Data object

Figure 3.22: Core notation elements of BPMN 
(events). These three objects are connected to each other via sequence flows, however only within the confinements of pools or lanes. Pools and lanes are constructs used to represent responsibilities in distributed business processes. They are discussed in more detail below. If a connection is made across pool boundaries, it is modeled using message flows, which we will expand on later.

A process consists of tasks. After starting a process (by means of an event), one task follows the other until the process ends (with an event). Tasks can be atomic (i.e., not refined further) or can contain sub-processes. In such cases, tasks are refined by an additional embedded BPD, which represents its detailed sequence of sub-tasks. This detailed sequence can be "hidden" and is represented by a "+" symbol at the bottom of the task.

A process begins with a start event and ends with an end event. BPMN offers a multitude of possibilities to define events that can trigger, complete or influence the course of a process. These will be discussed later.

At this point it is important to emphasize that a process can start with one or more start events and can end on any path through the process (see sequence flow and gateways below) with one or more end events. There must be a continuous sequence flow from each start event to at least one end event. Tasks, gateways or intermediate events must not be endpoints in the process and therefore always require at least one outgoing sequence flow.

A gateway represents a branch in the control flow. The exclusive (XOR) gateway requires a condition for each outgoing control flow, which according to the standard must always refer to the result of an immediately preceding task.

The parallel (AND) gateway tracks all outgoing control flows independently and in parallel. The branched control flows can be terminated separately with end events or explicitly merged again with another parallel gateway. After this merger, the control flow only continues once all incoming control flows have been completed (as with the split/join concept for activity diagrams).

The inclusive (OR) gateway can follow one or more paths, whereby a condition must be specified for path selection (as with the exclusive gateway). This condition must already be testable at the time of the decision, so the necessary data must have been generated in one of the previous tasks.

Decisions, which cannot be made on the basis of previously existing data, can be represented using the event-based gateway. This requires an event in each outgoing branch immediately after the gateway (e.g., an incoming message event or a timer event). When one of these events occurs, its respective branch (and only this branch) is activated. We will discuss this in more detail when discussing the use of events.

\subsubsection{Examples for Modeling Process Flows}

In order to work out the differences and similarities to the previously discussed modeling languages, we again make use of the examples already used above. 
Figure 3.23: Simple BPMN diagram

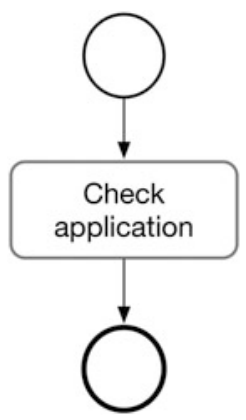

Figure 3.24: BPMN diagram with multipleoutcome decision

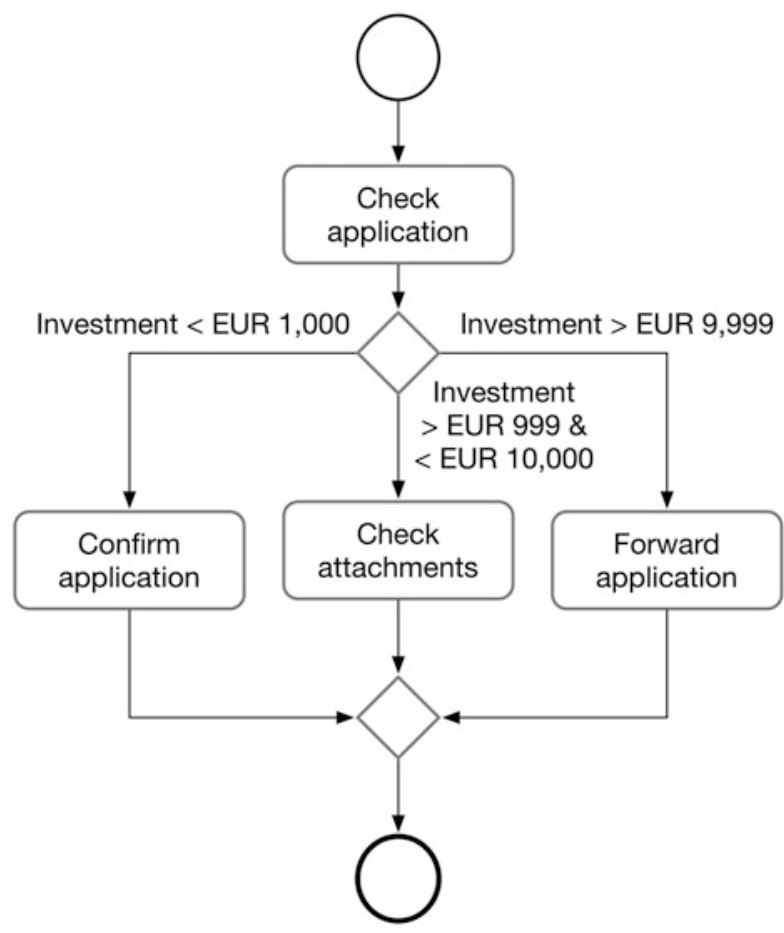

The example in Figure 3.23 shows a process with a single task in which an application (for which we have no detailed information here) is checked. The process ends after the checking of the application has been completed.

In the example in Figure 3.24, the process is extended by a decision with three possible results that are mutually exclusive. The application is checked, and the result of this check allows the making of a decision on how to further process the application. In BPMN, it is important that the data used as the basis for a decision is explicitly generated or received before the gateway.

Here, too, we can use branches to repeat parts of a process (cf., Figure 3.25). For this purpose, a gateway is inserted at the end of the part to be repeated and an 
Figure 3.25: BPMN

diagram with repeated

execution of process parts

(loop)

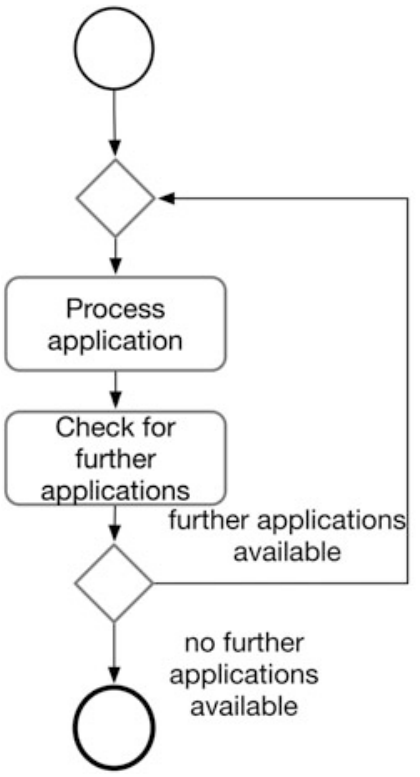

outgoing branch is returned to a closing gateway before the first task of the part to be repeated (a closing gateway is used for merging branches and has several incoming sequence flows and only one outgoing). The other outgoing branch of the opening gateway continues the process after the repetition is completed. The requirement to explicitly create the basis for decision-making prior to the gateway is illustrated here by the additional task of checking the existence of further applications.

The parallel gateway is used when two task sequences can be executed independently of each other (cf., Figure 3.26). The process modeled in the example therefore is only correct if the attachments can actually be checked independently of the application. If this is not the case, the two functions would have to be arranged sequentially. The merging gateway waits for both incoming branches to complete before continuing the process.

The example in Figure 3.27 shows the use of pools, lanes and data objects. The lanes are used to represent responsibilities in the process. With the BPMN elements introduced so far, we cannot map communication processes. The signals available in activity diagrams can therefore not be represented for the time being, which is why the example here is less specific than the version represented as an activity diagram. The use of message events, which we will introduce in a later chapter, will, however, remedy this deficiency. The assessment of the application is only transferred to the task "Reject application" in the case of a negative assessment as a data object (we can therefore assume that the rejection contains a substantive reason). In case the application is confirmed, the data object "Assessment" is no longer needed, so we can assume that in this case there will be no further justification. 


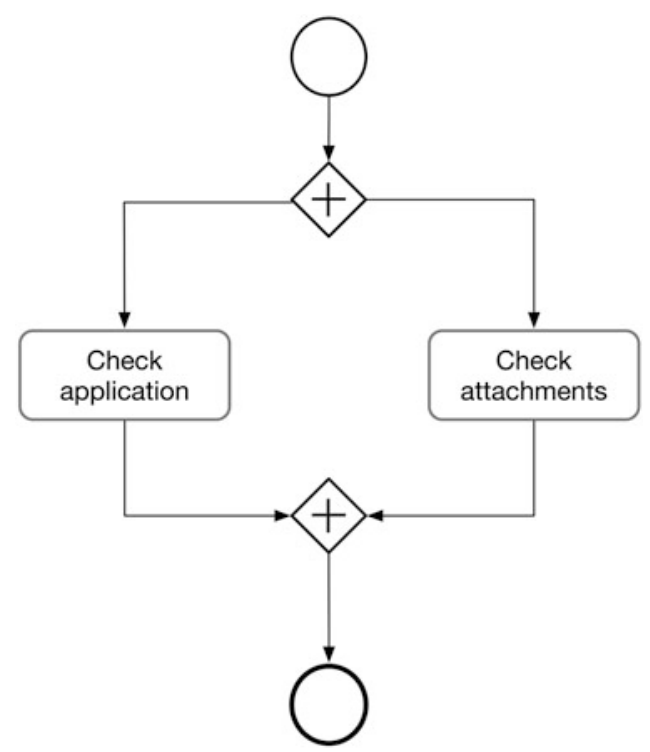

Figure 3.26: BPMN diagram with parallel execution of process parts

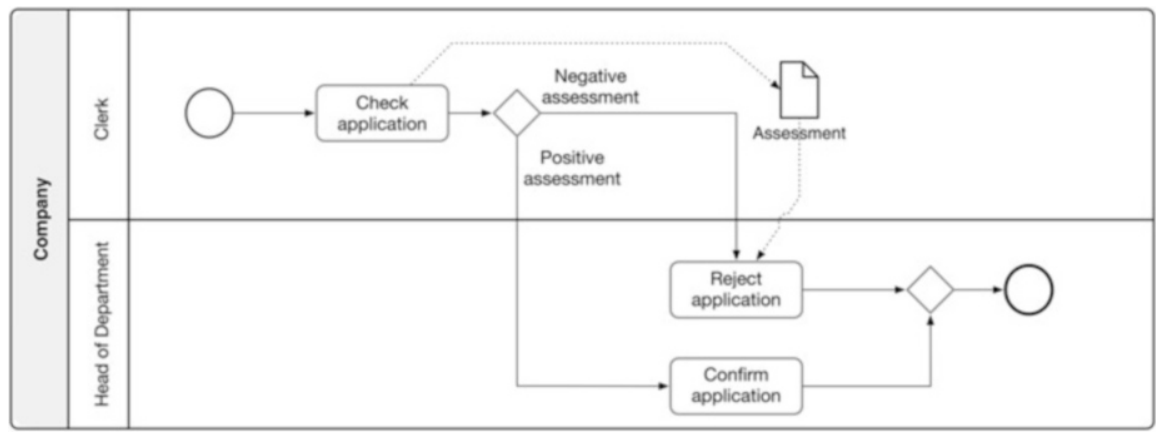

Figure 3.27: Example of BPMN diagram with pools, lanes and data objects

\subsubsection{Notation Elements for Controlling Sequence Flow with Events}

A distinguishing feature of BPMN is the very detailed and comprehensive set of event constructs, which enables exact control of the process flow.

Events indicate that something has happened and therefore represent points in times, as opposed to tasks that take a certain amount of time and effort to be completed. So far, we have only introduced start and end events. In the following we will describe start, intermediate and end events in detail once again (cf., Figure 3.28).

Events are always represented by a circle and usually an enclosed symbol. Simple circles indicate start events, double circle borders indicate intermediate events and thick circle outlines indicate end events. If no enclosed symbol is specified, the event 
Figure 3.28: Examples of different event types
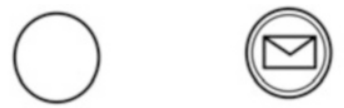

Start event Intermediate event
Figure 3.29: Basic BPMN triggers of start events
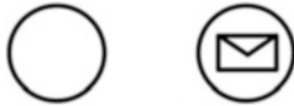

Application received

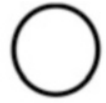

End event

Figure 3.30: Examples of combined or complex BPMN triggers for start events

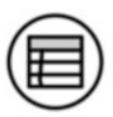

Utilization $>90 \%$

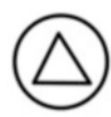

Onlinesystem down

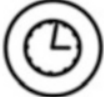

Application

Deadline

is of no particular type (blank) and can usually only be found at the start or end of a process or as a triggering intermediate event.

\section{Start Events}

Start events are used to trigger a process or subprocess. Generic (blank) events are often used if the trigger is either clear from the context, or when it is not yet known.

If a process is triggered on the basis of a particular point in time or a period of time or by a periodic event, the timer symbol (clock) is additionally used. A message symbol (letter) is used when an incoming message triggers the process (cf., Figure 3.29).

A process can also be triggered by a condition start event, if specific conditions need to be met in the process context in order to start it. Signal start events are used if some observable event from outside or inside the process should lead to the execution of a particular process. The pentagon as a symbol embedded in a start event indicates a combination of several potential start events, whereby only one of the events must occur to trigger the process (cf., Figure 3.30).

A process does not have to be limited to a single start event; processes can also have several alternative start events.

\section{End Events}

Processes always finish with end events, whereas the same symbols are used here as for the start events, with the exception of the timer symbol, the condition and the combination of parallel triggers. More specific end events include sending a message or issuing a signal to notify other processes about the end event at hand (for details, cf., section "Event Types").

One notable end event type is the termination end event (rightmost symbol in Figure 3.31), which terminates the entire process immediately, regardless of whether 


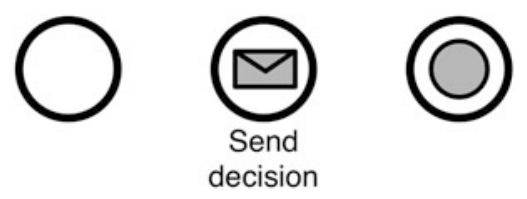

Figure 3.31: Examples of BPMN end events

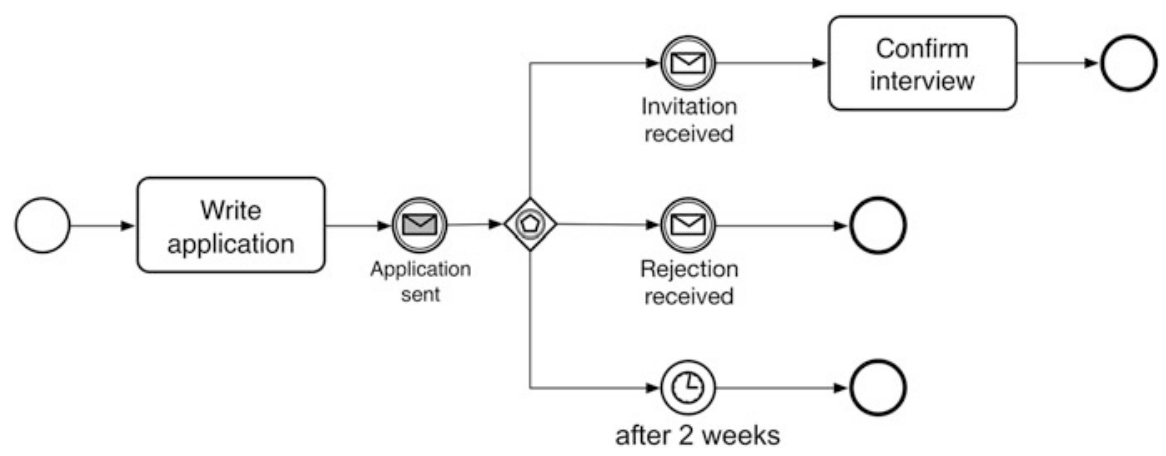

Figure 3.32: Example BPMN diagram showing the use of the event-based gateway

other sequence flows within the process are still running or not; that is, it terminates the entire process instance. A standard end event on the other hand always only terminates the process branch it concludes. Any further process branches that are still running will continue to be executed.

Processes can have several end events. A process without an end event is incomplete.

\section{Intermediate Events \& the Event-Based Gateway}

Intermediate events can be used anywhere in a process and are represented by a circle with a double border. They are used to represent intermediate results relevant for other processes, or if certain (external) events have an impact on the execution of the process at hand (for example, an incoming message or the expiration of a certain time period).

Gateways can also be event-based if the execution of a process is dependent on the occurrence of different events, which require different reactions and therefore follow different paths. Such dependencies can be modeled with an event-based gateway.

Figure 3.32 shows a process of applying for a job and waiting for different potential reactions. Depending on whether an invitation or a rejection is received, or whether a deadline of 2 weeks expires, different paths are taken in the process. The event-based gateway is the only gateway where the necessary information required to make a decision does not need to be available at the time the gateway is checked. An event-based gateway blocks the sequence flow until one of the downstream events is triggered, and then continues execution exclusively along the respective branch. 


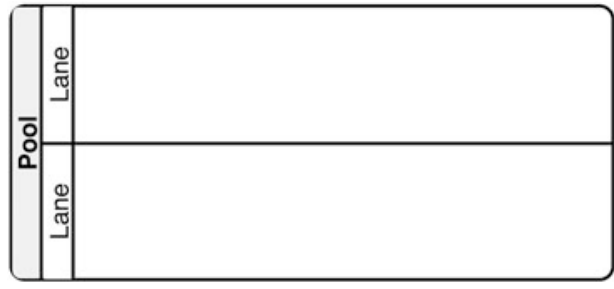

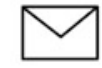

Message
Message

flow

Figure 3.33: BPMN notation elements for modeling communication

\subsubsection{Notation Elements for Modeling Communication}

BPMN also enables the modeling of distributed business processes. Although BPMN clearly focuses on the process flow during modeling (similar to flowcharts, EPCs or activity diagrams), it also enables the structuring of the process in accordance with the participants involved and their associated responsibilities. The modeling elements available for this purpose are described in this section (cf., Figure 3.33).

A pool represents a company or an organizational unit in a company, such as a department. Each (swim) lane in a pool represents a person or role involved in the process that is assigned to this pool.

BPMN allows the representation of the interaction of two or more processes. The aforementioned pools and lanes are necessary for the representation of collaborations. Separate lanes are required for all persons or groups involved in a process, and separate pools are necessary for each process or organizational unit that is responsible for this process. Each pool thus contains its distinct processes with separate start and end events. Nevertheless, these individual processes can strongly influence each other, in which case they are coupled via message flows.

Message flows indicate that data is exchanged between different processes. Therefore, no message flow can take place within a process (pool). Consequently, there are no message flows within a lane or between different lanes of a single pool. Sequence flows show which activities are executed in which order and do not explicitly constitute an exchange of data. In contrast to message flows, they may only be used within a pool and not between different processes (pools).

Message flows can be augmented with message elements, which are used to explicitly represent the exchanged data and contain a more precise specification of the transmitted information.

Message flows can be used in different ways: they may either originate from pools and activities, and also end there, or they can be explicitly sent by send message events and received by receive message events. The first case is useful for the descriptive modeling of business processes in which a communication process is to be represented that does not necessarily have to be described exactly. A message originating from an activity or pool is sent at some point during task or process execution - the exact time remains unclear. A message ending at a pool only states that the represented organization receives this message, but not which activity 


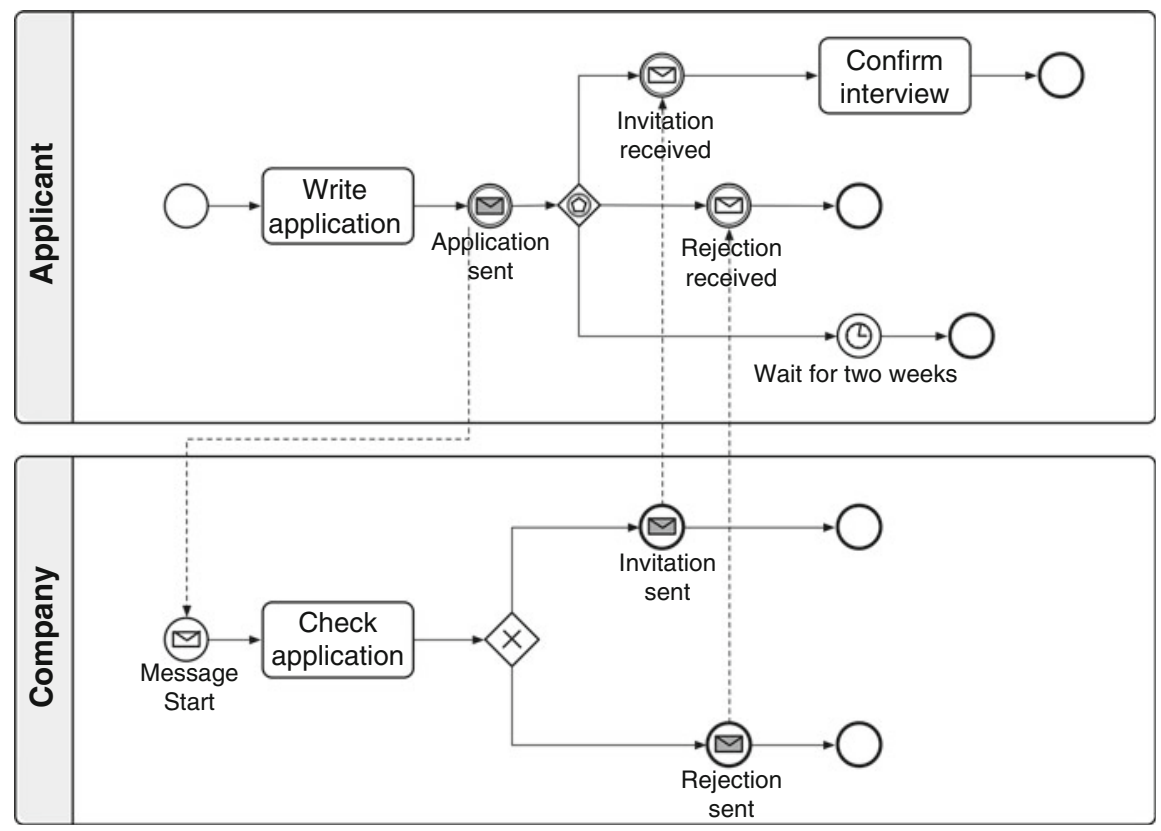

Figure 3.34: Example BPMN diagram showing communication-oriented processes

it triggers or how it is handled within a process. This can be useful when modeling external organizational units, whose detailed behavior is unknown. An exact specification of communication processes, however, is only possible by using explicit send and receive events.

\subsubsection{Examples for Modeling Communication-Oriented Processes}

In the following, we extend the example that we used to demonstrate the use of events with the communication partner not depicted originally, i.e., we now also model the process of the company to which an application is addressed.

The example in Figure 3.34 shows two processes (one per pool) that are linked by message flows. The company's process is triggered by an incoming application, which is represented here in the first message flow. After checking the application, the decision can be made whether to send an invitation or reject the application. In the upper pool, the applicant waits for an answer for a maximum of 2 weeks (as represented by the intermediate timer event). The event-based gateway activates the process branch whose event occurs first. The related send and receive events are linked via message flows. It is important to note here that message flows always represent 1:1 relationships - that is, a sent message can be received exactly once and a receive event can react to exactly one message. 


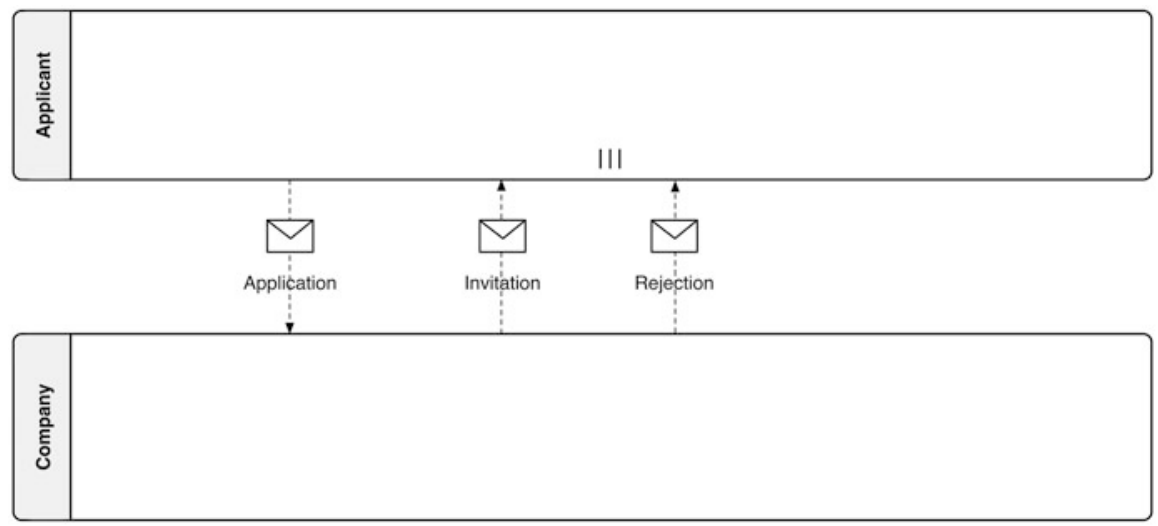

Figure 3.35: Example BPMN diagram showing the exclusive use of communication interactions

The example in Figure 3.35 shows how BPMN can be used to only represent communication acts between the actors in a distributed process. The pools are used here as black boxes, i.e., the behavior contained in them is not shown and remains unknown. All we see is that messages are exchanged, but the order of the messages is not defined. Since the specified events for sending and receiving are missing here, we augment the model with message elements attached to the message flows in order to be able to comprehensibly describe the nature of communication. Another extension of the original process model is constituted by the modifier in the upper pool, which indicates parallel multiple execution of the process contained in the upper pool. This means that the process in the lower pool could or even must be able to handle several applications arriving in parallel and independently of each other.

Empty and filled pools can also be combined as required. If, for example, we wanted to represent the process of handling an incoming application, we could leave the pool "Applicant" unspecific, since we do not know the behavior of applicants (nor is it relevant), but need to know that we can receive an application from them and that we will direct our responses to them again eventually.

\subsubsection{Notation Elements for Modeling Complex Business Situations}

The notation elements of BPMN introduced so far enable the representation of business processes from the point of view of the participating organizational units. BPMN allows keeping process models vague or leaving parts of them unspecified if they do not seem relevant for the objective of modeling. In some cases, however, a process needs to be defined as precisely as possible and represented in all its variants, covering all possible exceptions. This is necessary, for example, if the model is intended to serve as the basis for IT-support of the work processes depicted. If aspects are omitted or abridged, the result is a discrepancy between the real work process and the support measures developed based on the model, which ultimately 

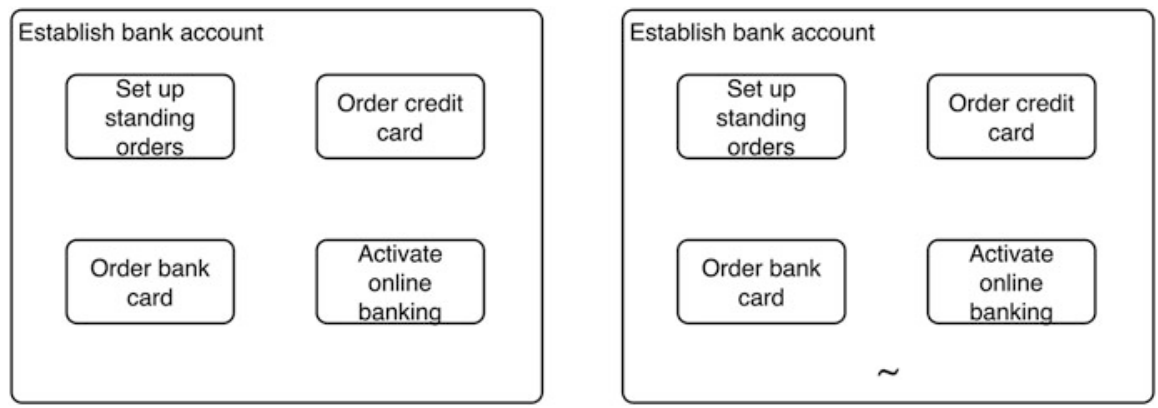

Figure 3.36: Parallel and ad-hoc subprocesses

would lead to unsatisfactory tools and work-arounds. This section describes the BPMN notation elements that enable more complex and comprehensive process descriptions. Due to the variety of scenarios that can be represented, examples are given here directly with the descriptions of the respective elements.

\section{Variants of Activity Modeling}

In the following section, special features for the general modeling of activities, as well as for the modeling of activities as subprocesses are explained in more detail.

\section{Subprocesses}

Processes can include detailed specification of tasks via subprocesses. This method is mostly used to maintain a comprehensive overview of a process when creating large, extensive models, while still being able to specify detailed task descriptions. Subprocesses can be collapsed to tasks, which are then shown in the overall process with a small plus sign. If appropriate tool support is available, collapsed tasks can be dynamically extended again to view the detailed subprocess specifying the exact execution of the task.

Subprocesses also can be used to combine several tasks in a single execution context without specifying their exact sequence. The model on the right (an ad hoc subprocess) in Figure 3.36 only indicates that any number of the embedded activities can be performed but makes no statement about their relationships. The left model specifies that all four embedded tasks must be executed before the subprocess is completed. It makes no assertion about their order or other relationships - the activities can be carried out in parallel or in any sequence.

\section{Types of Tasks}

Task types describe the character of a task in more detail, indicating for example whether it requires human involvement or can be executed automatically in an IT system. Modifiers as shown in Figure 3.37 are used to distinguish between service tasks, receive tasks, send tasks, user tasks, business rule tasks, script tasks, and manual tasks (illustration from top left to bottom right). These modifiers do not 

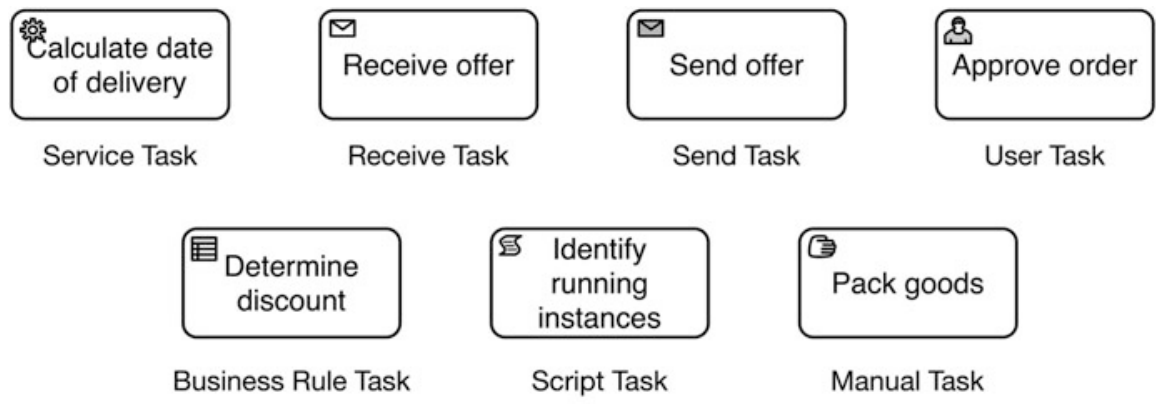

Figure 3.37: Different task types

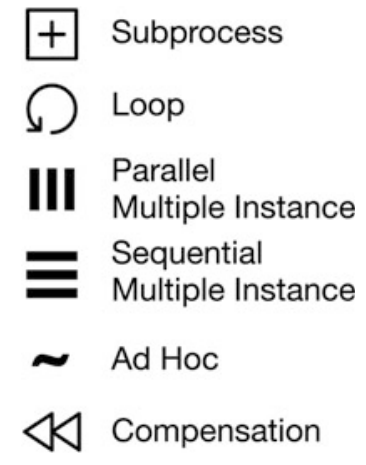

Figure 3.38: Task modifiers for diverse behavior specification

necessarily have to be used, but they do specify the semantics of a process model in more detail.

\section{Execution Behavior of Tasks}

Tasks can have different markers that describe their execution behavior. Single tasks, entire processes or subprocesses can be executed several times in loops, in parallel, sequentially, or can be marked as ad hoc tasks or as compensation tasks (cf., Figure 3.38).

For a looped task or process, a termination condition can be specified in addition to the symbol. If the termination condition is reached, the task or process in question is no longer executed and the superordinate process is continued. If a task can be executed several times in parallel, this is indicated by three vertical lines. For example, the "check application" task could be carried out by several agents for several received application documents. If parallel execution is not possible, but the individual cases are still independent of each other, the sequential multiple instance marker is used, which is indicated by three horizontal lines.

With ad hoc tasks, the exact sequence of the sub-tasks contained in the task is unknown a priori and is selected during the execution of the process. It is also 


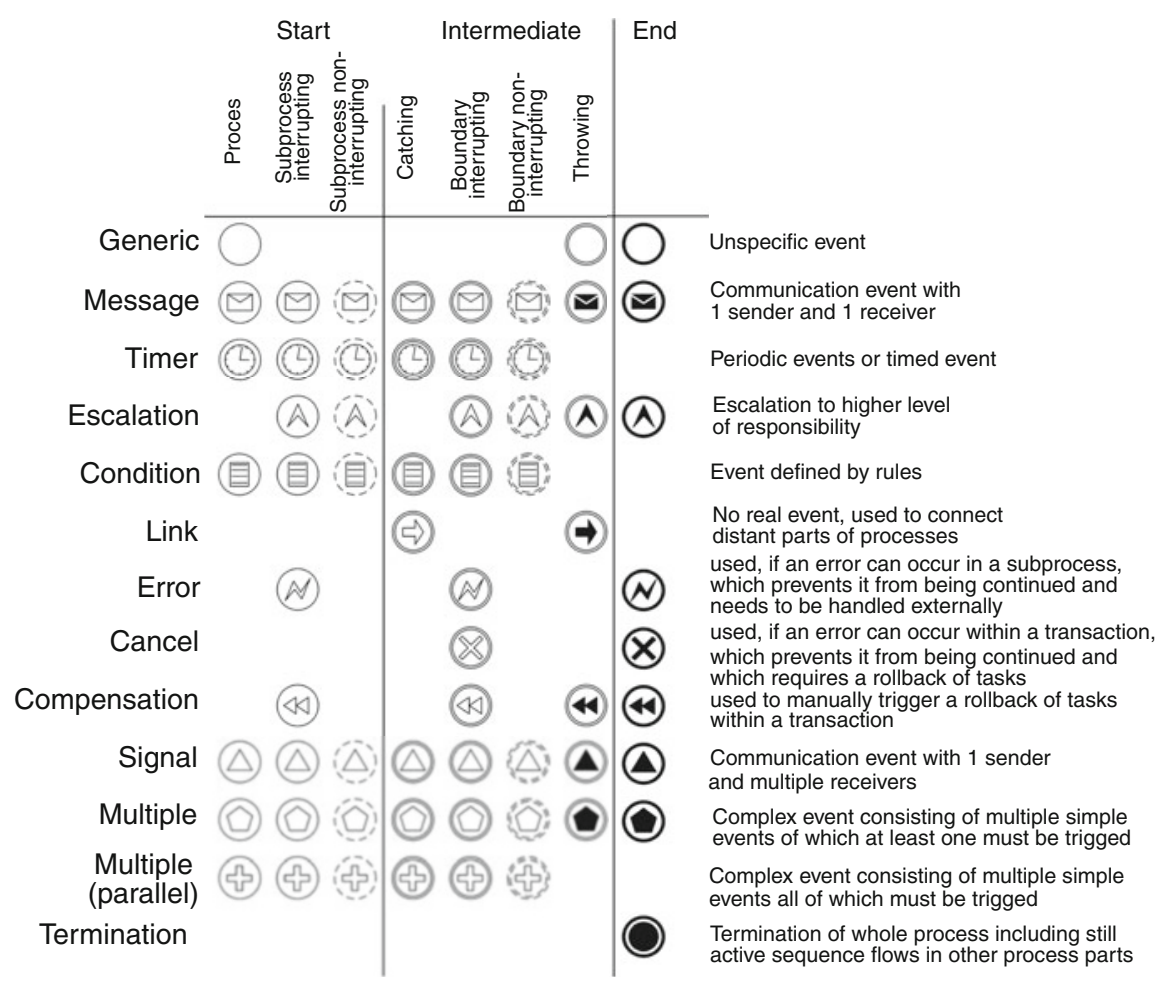

Figure 3.39: Different BPMN event types

possible to omit some of the sub-tasks and only execute those that are required in the specific situation. Such processes are indicated by a tilde (as shown above).

Compensation activities are used in transaction modeling and are described below.

\section{Event Types}

BPMN offers a large number of different events targeting different areas of application for detailed process control. While we will deal with these in detail in the following, an initial overview is provided in Figure 3.39 to show the underlying structure of event types.

In general, events can occur in three different variants, which we have already introduced above:

- Start events trigger new process instances, that is, they start the execution of a process. They are always "receiving" in nature, i.e., they react to stimuli from outside (e.g., incoming messages, time sequences, etc.). 
- End events are events that are triggered when a process instance is finished. They are always "sending" in nature, thus potentially provide stimuli for other processes or process parts.

- Intermediate events occur within the sequence flow, i.e., they have both an incoming and an outgoing flow (exception: link event, see below).

Start events can also be differentiated according to their surrounding modeling element. They can be used to either trigger an entire process or to trigger subprocesses. The second case is called an "event subprocess" and can be specified as an "interrupting" or a "non-interrupting" form. An "interrupting" start event indicates that the control flow is completely transferred to the subprocess, i.e., all other task within the respective pool are interrupted and cannot be continued. "Noninterrupting" event subprocesses are started when the respective event occurs without interrupting the execution of the task currently running within the pool the subprocess is placed in. This can be used, for example, to react to events that should not or cannot be handled in the main process of a pool, but whose occurrence should result in a reaction without affecting the main process (such as customer inquiries about the status of an order processing while the order is being processed in the main process).

Intermediate events basically exist in "receiving" and "sending" variants. The "receiving" variants (occurring event) block the sequence flow until the specified event arrives. The process therefore cannot be continued until the event has occurred. The "sending" variant (triggered event) indicates the occurrence of certain events in the course of executing a process (or also an occurrence between processes from different pools). Events often are used reciprocally in comprehensively modeled processes, i.e., a receive event exists for each send event.

Receiving intermediate events also exist in a "boundary" form. These events are "pinned" to tasks (i.e., are graphically attached to the (lower) boundary of the task) and indicate that it is possible to react on the respective event during the execution of the task. The reaction is specified by a sequence flow originating from the attached event, which leads to the respective tasks to be performed. The boundary intermediate events in general (with some exceptions) again exist in an interrupting and a non-interrupting form. The interrupting form stops the execution of the task marked in this way and continues the sequence flow exclusively via the attached event. The non-interrupting form allows the further execution of the task marked in this way, and the sequence flow originating from the event is triggered in parallel.

The triggers that lead to the occurrence of boundary events can come from outside the tasks (for example, incoming messages from other pools) or also from within the task, provided that these triggers are detailed by a subprocess. For example, an error in the execution of a subprocess can lead to activities in the main process via an interrupting error boundary event (such as documenting the error and escalating it to superiors).

We have already used message and timer events in basic BPMN modeling. We will now consider the other event types in the context of their respective application areas. 

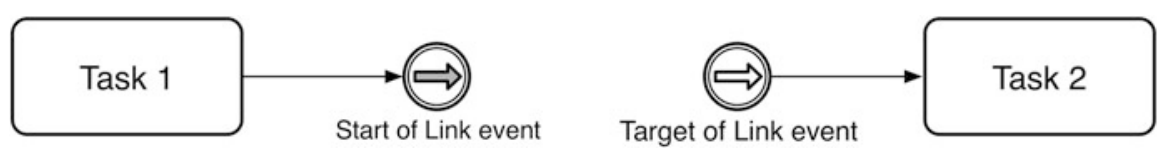

Figure 3.40: Link event

\section{The Link Event}

For more complex or extensive processes, tracking sequence flows through the diagram can sometimes be difficult. Sequence flows crossing each other or sequence flows with many changes of direction are difficult to read and are detrimental to comprehensibility and clarity.

In such cases, the link event can be used (cf., Figure 3.40). In contrast to the other events, it semantically does not represent a real event, but merely serves as a connector between two sequence flows that are far apart. The coupling is carried out via the designation of the sending and the receiving event. There must always be a 1:1 assignment (implicit parallelization by one triggering and several receiving link events of the same name is therefore not permitted).

Link events should only be used in cases that cannot be resolved in any other way in order to increase clarity, since the effort involved in searching for related link events can even exceed the tracking of complex sequence flows (as long as there is no tool support for jumping to or visually marking related events). Choosing an alternative arrangement of activities or lanes is usually the better choice.

\section{Use of Signals}

In BPMN, messages can only be used for communication between pools. In addition, message-based communication always has exactly two endpoints, so it can only connect exactly one sender to exactly one receiver at a time. If information is to be made available globally within a collaborative process and this is to happen independently of pool boundaries, signals can be used. Signals can be triggered in a process (as intermediate or end events) and are then available both within the pool and in all other pools of the same collaboration.

Signals can be used, for instance, to inform all pools of a collaboration about the termination of one of the represented processes. This means that all other processes that are still running can complete their processes cleanly and there are no dangling processes left that can no longer be completed, e.g., because an expected incoming message no longer arrives due to an aborted process of a communication partner.

\section{Handling of Exceptions and Interruptions}

Activities, i.e., tasks and subprocesses, can be aborted or interrupted by certain events. This is indicated by event symbols attached to the respective task. Two solid outer circular lines in the event element indicate that the task is interrupted by the event; two dashed circular lines indicate that the task is not interrupted but can be continued while simultaneously reacting on the exception that occurred. In the example in Figure 3.41, we react on a deadline, i.e., we model that the execution 


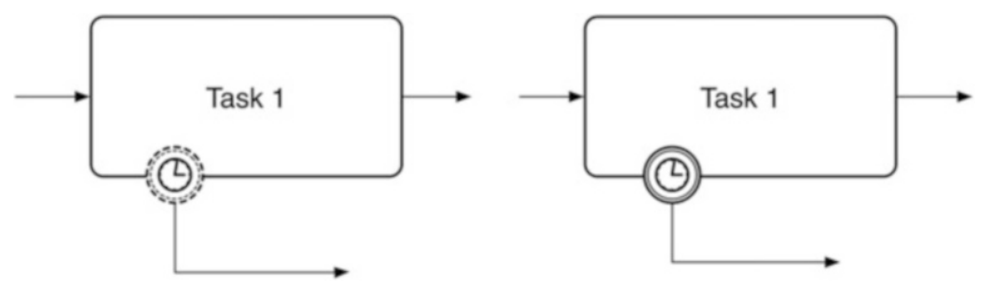

Figure 3.41: Non-interrupting and interrupting boundary events

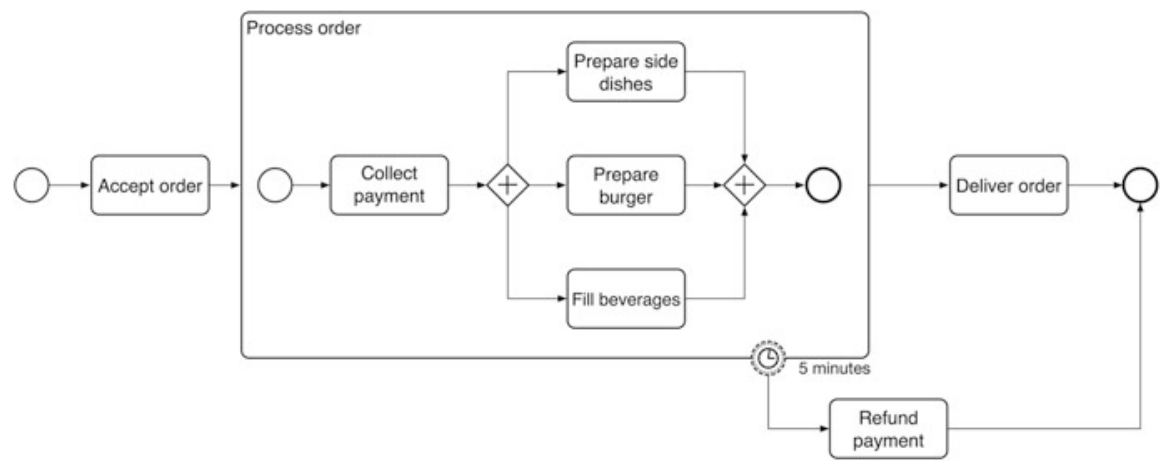

Figure 3.42: Example for use of a non-interrupting timer event

of the task must not take longer than a certain time span (on the left) or requires some reaction if it lasts longer than a certain time span (on the right). In either case, the model must contain information on what has to happen when task execution takes longer than anticipated. This information is modeled as a sequence flow emanating from the attached boundary event. Reactions to unforeseen triggers such as errors or escalations can also be modeled and displayed in the same way using the respective event type.

\section{Example: Non-interrupting Timer Events}

Figure 3.42 shows a subprocess that includes the activities of processing an order in a fast food restaurant, which should not take longer than 5 minutes. If the processing of the order takes longer than 5 minutes, the customer should receive their money back. The order should still be completely processed. If we had used an interrupting boundary event here, the customer would only get their money back, but not their order.

\section{Different Ways of Terminating Processes}

Sequence flows in a process are usually concluded with an end event. Such end events, however, only terminate the execution of the respective sequence flow. If other sequence flows are active in parallel (for example, because they were opened 
by a parallel or inclusive gateway or because they were triggered by boundary events), their execution will continue to be carried out. There are several ways to terminate a (sub)process completely and immediately (i.e., terminate execution in all branches).

\section{The Terminate Event}

The terminate event aborts all active branches of a process within a pool immediately. Processes in other pools are not affected and should therefore be informed of the termination by sending a signal before the termination, if necessary (e.g., if there is the risk of waiting for further input from an already terminated process instance).

\section{The Error Event and the Escalation Event}

The error event semantically indicates the occurrence of an unforeseen error in process execution and is usually used for subprocesses. It immediately terminates the execution of the whole subprocess. The reason for the error can be given to the event as a parameter. Receiving error events can be attached to the subprocess as a boundary event for the enclosing task element to react to these errors in the superordinate process and trigger corresponding activities. Attached error events are always interrupting, i.e., they terminate the execution of the subprocess (including all active sequence flows in branches in which no error occurred). As a "weaker" variant, the "escalation event" can also be used in an identical way. The escalation event also is available in a non-interrupting form and thus allows the continuation of the execution of the subprocess in which the problem occurred.

If the effects of activities already performed have to be reversed when subprocesses are terminated, the transaction handling mechanism and constructs provided in BPMN can be used. They are described in the next section.

\section{Transactions}

BPMN also offers the option of representing transactions in a process. A transaction is a set of tasks that is to be executed as a whole, either completely or not at all. In particular, if a task fails, the effects of other already completed tasks need to be reversed. BPMN introduces the concept of transactional subprocesses in combination with compensation events and tasks. Compensation tasks roll back the effects of process steps that have already been executed by means of countermeasures which are initiated in a further process step.

In a transaction subprocess (characterized in BPMN by a double border of the enclosing task element), each task is assigned a compensation task via a boundary compensation intermediate event (indicated by a "rewind" symbol). If the transaction is aborted or should explicitly be undone retroactively, the respective compensation task is executed for each task that has already been successfully completed. The abort end event (marked by an " $\mathrm{X}$ ") can be used to abort a transaction while it is still being carried out. As an end event in a transaction subprocess, it causes its immediate termination and triggers the compensation tasks. When attached to the transaction task as a receiving intermediate boundary event, it determines the further course of the process after the transaction is terminated. As a result of the concept of 


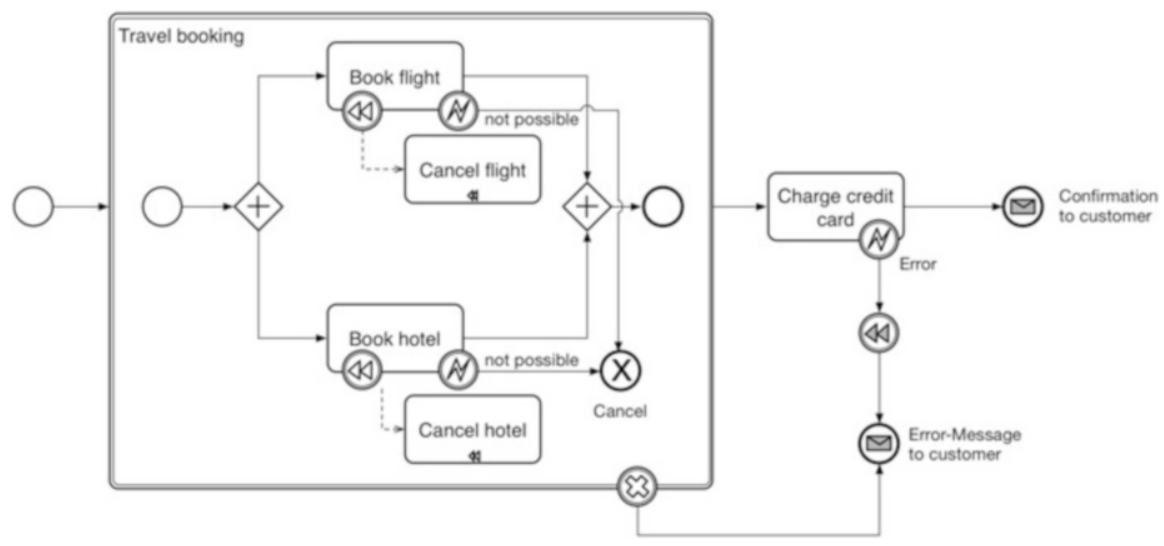

Figure 3.43: Exampe for transaction subprocesses and compensation events

compensation, transactions can also be rolled back after they have been successfully completed. Outside the transaction, a sending intermediate compensation event can be used to retrospectively trigger the compensation tasks contained in the referenced transaction.

Figure 3.43 illustrates these concepts using a travel booking process.

A travel booking consists of a flight booking and a hotel booking that can potentially be done in parallel. If one of the bookings is not possible, the other booking must be canceled, if it has already been done. An error in one of the bookings leads to the transaction being canceled (triggering the termination event) and leads to sending an error message to the customer. Outside the actual transaction, an error in charging the credit card leads to cancellation of the entire booking by triggering the compensation tasks retrospectively.

\section{Event-Triggered Subprocesses}

Event-triggered subprocesses are an alternative to boundary events when handling non-standard incidents that might occur in (sub)processes. While boundary events attached to subprocesses lead to the reaction to such incidents in the superordinate process, strictly local reactions (i.e., reactions that do not have any implications for the overall process) can be kept in the context of the subprocess by using eventdriven subprocesses.

Figure 3.44 shows an example of a timer-controlled non-interrupting subprocess. It picks up on the scenario already used above to demonstrate non-interrupting boundary events and shows the process of preparing an order in a fast food restaurant.

Event-triggered subprocesses can be started with the same types of events that are available as boundary events, both in their interrupting and non-interrupting versions. Semantically, as already mentioned above, they differ only in the way the incident triggering the event is handled - locally within the subprocess or externally within the superordinate process. Depending on the process, one or the 


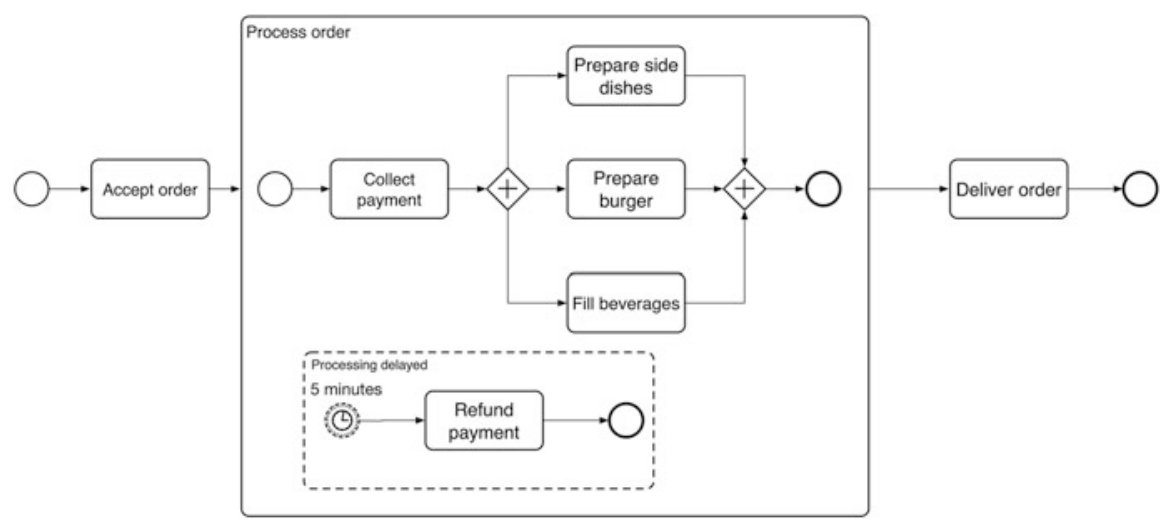

Figure 3.44: Example for non-interrupting event-triggered subprocesses

other variant can lead to a more meaningful and/or comprehensible form of representation.

\subsubsection{Choreography Diagrams}

The ability to explicitly model the interplay of actors in a collaborative process has been introduced in BPMN 2.0 in the form of choreography diagrams. A choreography depicts the process of exchanging messages between different actors. It thus provides a different view on a collaboration, focusing on the sequence of the transmitted messages independently of the processes of the individual actors.

Although a representation of communication is possible in BPMN process diagrams by means of collapsed pools and the messages exchanged, the exact sequence, conditional message flows or loops cannot be represented in this way. For instance, the example of an application process used to demonstrate the use of collapsed pools as shown in Figure 3.35 does not include information on whether the invitation message and the rejection message are mutually exclusive or can occur in parallel. This can be visualized with a choreography diagram.

Figure 3.45 shows the choreography representation of the application process shown above as a collaboration process. Here, the process of message exchange is in the focus of representation. Choreography tasks represent the exchange of one or more messages between two or more partners. In their simplest case they correspond to sending a single message from one partner to another.

Each choreography task is triggered by one of the partners involved by sending the first message. This triggering partner is entered in a box with a light-colored background at the upper or lower edge of the choreography activity. The names of the other party or parties involved are entered into boxes with darker backgrounds on the other border of the task. Which partner is entered at the top and at the bottom is at the discretion of the modeler. Usually, if there are several choreography tasks 


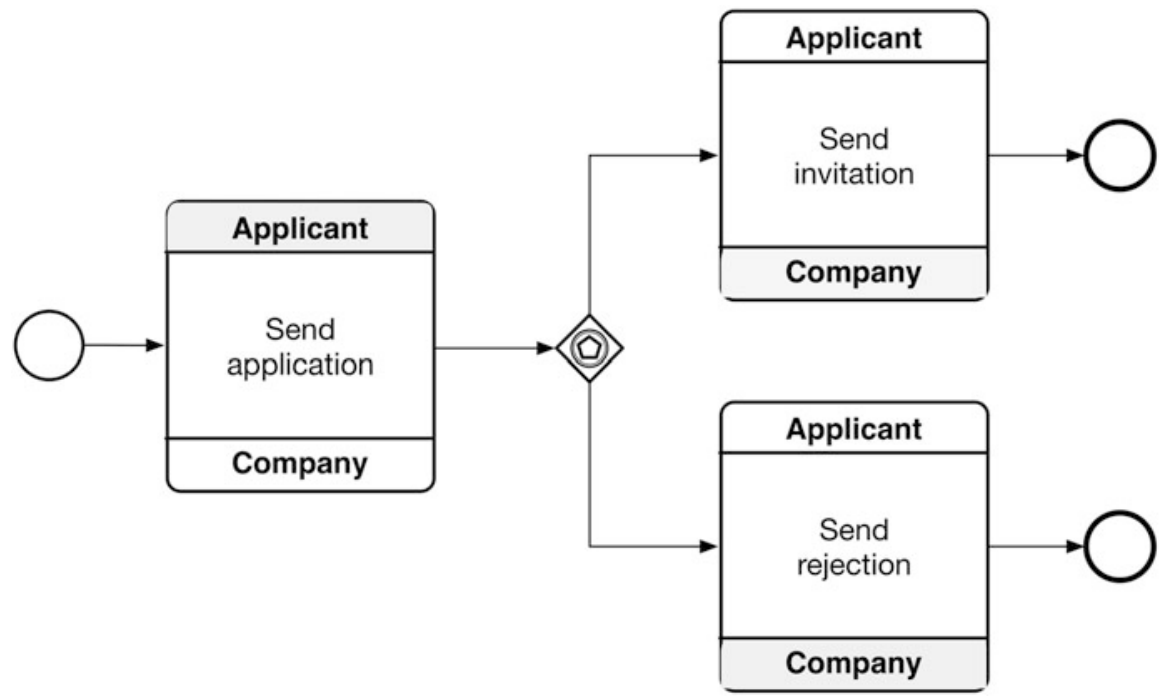

Figure 3.45: Example of a choreography diagram

between the same partners, the arrangement will remain identical to allow for better comprehensibility. If corresponding collaboration diagrams are modeled, it is recommendable to use the vertical arrangement of the pools as a basis for labeling the partners in the choreography tasks.

Choreography activities with more than two partners do not occur in the example shown above. In case more than two partners would be involved, several partner fields can be added at the top or bottom. However, only one field can have a lightcolored background, since only one of the partners initiates message exchange with an initial message.

A sequence flow is defined in the choreography diagram between the choreography tasks. Modeling the sequence flow in choreography diagrams essentially corresponds to the sequence flow modeling of ordinary BPMN processes. However, certain elements of process modeling do not make sense in connection with choreography modeling and are therefore not permitted. For example, there are no message events within a sequence flow, since the message exchange is, by definition, part of the choreography tasks. Accordingly, in the diagram above, event-based gateways are not followed by events, but rather by choreography tasks. The path is selected for which the associated choreography task is first started by the respective triggering message.

If one wants to know which messages are exchanged in each choreography task, these can be added to the diagram in the form of letter symbols which are linked to the respective partner sending the message. The letters are color coded in the same way as partner fields. A letter symbol with a light-colored background represents the message with which a choreography task is triggered. The letter symbols of the other messages are displayed with darker backgrounds. 


\subsubsection{Classification}

In recent years, BPMN has advanced to be the standard choice for modeling business processes in industrial practice. Its comprehensive set of language elements makes it suitable for many application areas, from documentation to the automationsupported execution of business processes in organizations. The extensive vocabulary can at the same time be seen as a shortcoming of BPMN due to the increased complexity of the notation. In particular, the large number of event types with semantics that are sometimes hard to distinguish leads to increased effort when learning the language.

Potential issues of comprehensibility of the models when using the full set of notation elements is usually countered by using a reduced set of elements in suitable cases. For the descriptive documentation of business processes, it is usually not necessary to use the complete set of events and more complex task types. Only when a process model is to be validated or executed, for example by simulation, is it necessary to enrich the models with information on non-standard cases or exceptions. In such cases, the simpler models can be used as a basis for supplementation.

BPMN is one of few Business Process Modeling languages that explicitly deals with communication processes between participating actors, and enables the modeling of the same. During the development of the language, however, the starting point for specifying communication flows was the coupling of technically distributed information systems. BPMN implicitly assumes that within a pool (i.e., between lanes) it is not necessary to explicitly represent communication between actors, because they all have access to the same information infrastructure. Message flows are only modeled between pools. They are used during execution to describe the mapping of the data structures used in the source pool to those of the target pool. A message flow therefore essentially corresponds to a data transfer from one information system to another and therefore always represents a communication process with exactly one sender and exactly one recipient - several recipients cannot be addressed with a single message. While this mechanism can also be used to represent non-technical communication, its expressiveness is limited. In particular, communication between two or more actors without clearly definable messages can only be modeled in non-standard-compliant and ambiguous ways. This limitation is owed to the claim of the executability of the created processes and also exists in other communication-oriented approaches.

In addition, BPMN focuses on processes with a fully specifiable control flow. It reaches its limits when process parts are strongly case-specific and cannot be described in detail in advance. In recent years, different approaches have emerged for such processes, which either adopt a declarative modeling approach for representing the execution conditions of process parts or focus on the communication processes between the actors involved. As an example for the latter category, we introduce Subject-oriented Business Process Modeling (S-BPM) in the next section. 


\subsection{S-BPM}

In contrast to other modeling approaches, subject-oriented process modeling describes business processes primarily from the point of view of communicating actors or systems.

When modeling according to the subject-oriented approach, the subjects as representatives for those involved in a process are the focus and starting point of representation. It essentially describes who communicates with whom in which form and how the individual actors react to received messages. Communication is described by defining the messages that are exchanged between the subjects. The behavior of the subjects is described separately by state diagrams, whereby three different state types are used. A subject can wait for a message, send a message, or do something without communicating with other subjects. The latter state type is called the function state and is used to describe the actual behavior, that is, the activities of a subject.

\subsubsection{Notation Elements}

When modeling according to the subject-oriented approach, the subjects as representatives for those involved in a process are at the center of attention for modeling. The modeling of a process essentially takes place in two stages with an increasing level of detail. First the interaction diagram is created, in which the subjects and their message exchange are modeled. In a further stage, the behavior of each subject is described in a separate behavior diagram.

For the interaction diagram (cf., Figure 3.46), the subjects involved in a process are defined first. A subject is an active entity but does not necessarily have to be a human actor. Technical systems can also be subjects, as long as they play an active role in the process. Subjects must always be described abstractly, i.e., not for specific persons or machines, but on the basis of the necessary tasks to be fulfilled in the process (e.g., "application examiner" and not "Mr. Miller").

Messages are exchanged between the subjects. The interaction diagram only defines which messages exist and who sends and receives them. The order of the messages is not defined here.

For each subject, a behavior diagram describes the order in which it sends and receives messages or executes functions (cf., Figure 3.47). The individual states are

Figure 3.46: Notation of S-BPM interaction diagrams

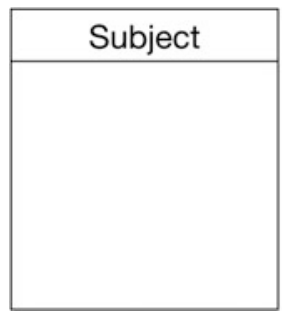




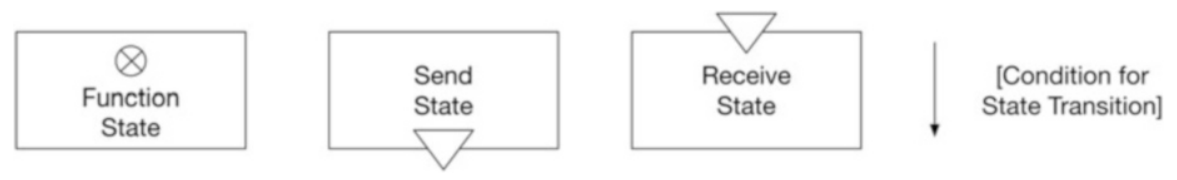

Figure 3.47: Notation of S-BPM behavior diagrams

related to each other by connections which describe the conditions of the transition from one state to the next. Their use depends on the type of condition used:

For each function state, what is to be done in the respective behavior step is described. The end conditions of a function state correspond to the outgoing connections that emanate from the respective function state. If the function can lead to different results, different subsequent states can be activated via different transition conditions. This enables the representation of alternative behavior patterns.

In a send state, a message is transmitted to a recipient. The subject remains in the state until the recipient is able to receive the message. Who the recipient of the message is and which message is transmitted is described at the outgoing connection of the send state.

The respective subject remains in a receive state until one of the messages that the receive state can accept has arrived. Since different messages can be accepted in any particular receive state, different subsequent states can be activated depending on the type of message received. For this purpose, several outgoing connections can be used to describe which message from which sender leads to the corresponding state transition. In this way, it is also possible to react differently to the same message from different senders.

\subsubsection{Examples}

In order to work out the differences and similarities to the previously discussed modeling languages, we again make use of the examples already used above. In the first example there is no communication, therefore we focus on the behavior diagram of the only subject involved.

The example in Figure 3.48 shows a process with a single task in which an application (for which we have no further information here) is processed. The process ends after the processing of the application has been completed. A behavior diagram must always have a start state, which is marked by a triangle in the upper left corner. There must also be an end state, marked by a triangle in the lower right corner. To fully describe the behavior of the subject, we need a state transition that identifies under what conditions the state "Check application" can be left. Therefore, we insert a state "Done" here, which we mark as the final state and which does not contain any expected activities.

In Figure 3.49, the process is extended by a decision with three possible results that are mutually exclusive. The application is checked, and the result of this 


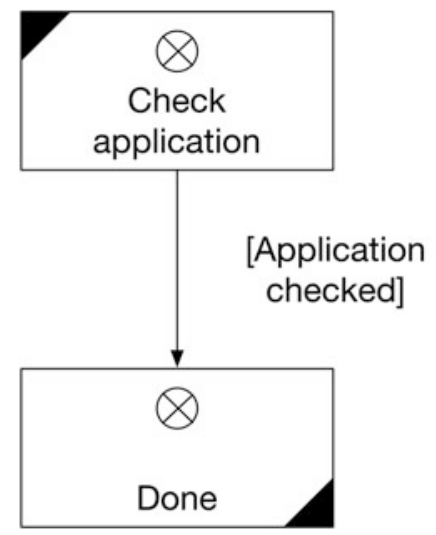

Figure 3.48: Simple S-BPM behavior diagram

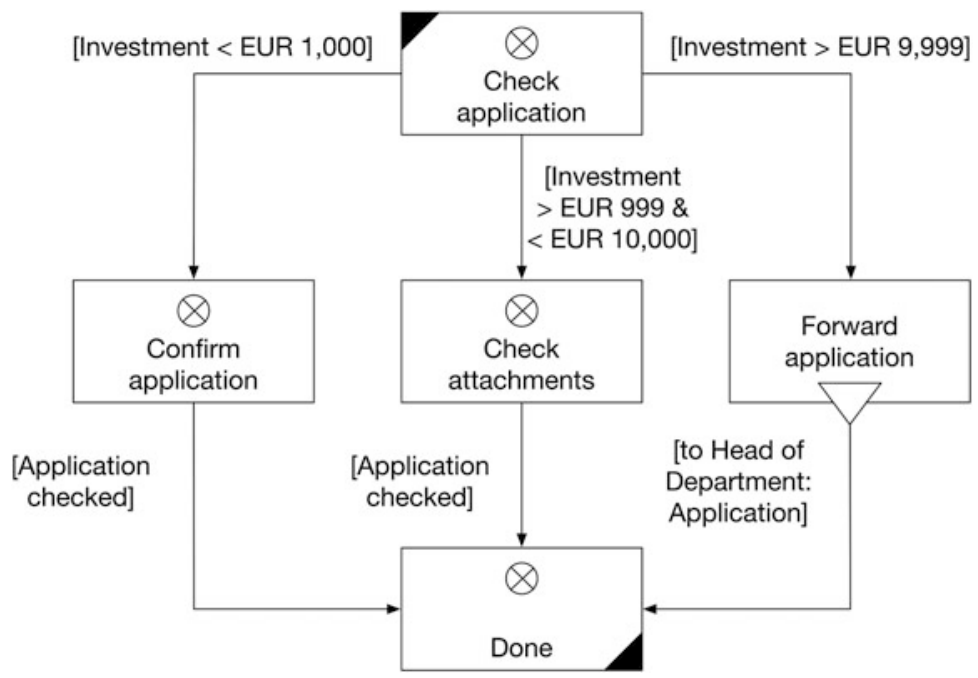

Figure 3.49: S-BPM behavior diagram with a multiple-outcome decision

examination allows a decision to be taken on further processing. In the case of an investment sum of EUR 10,000 or more, the application will be forwarded. We indicate this by a send state and specify at the outgoing connection who is to receive the request. For the process to be fully specified, a behavior diagram for the head of department would also have to be created at this point.

S-BPM also allows the execution of parts of a process repeatedly. For this purpose, a connection is inserted at the end of the part to be repeated, provided with a repetition condition and returned to the first state of the part to be repeated 


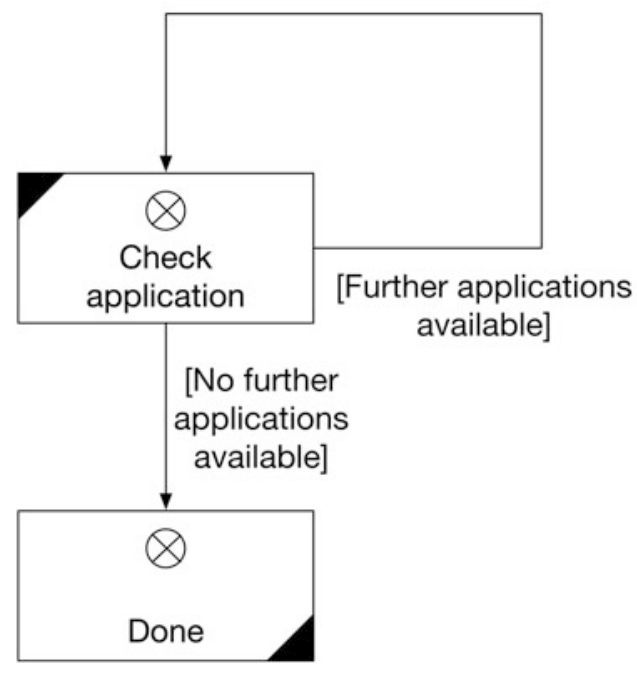

Figure 3.50: S-BPM behavior diagram with repeated execution of process parts

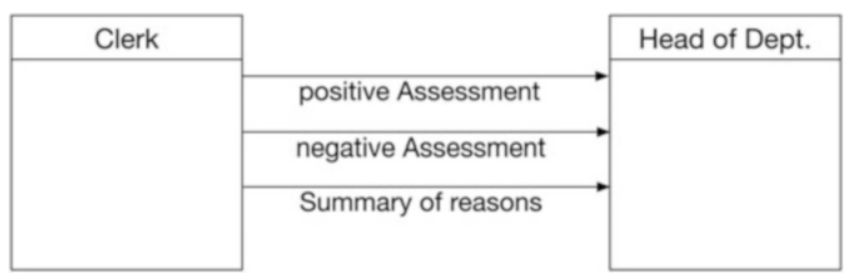

Figure 3.51: Example of S-BPM interaction diagram

(cf., Figure 3.50). The other outgoing connection continues the process after repetition completion.

The example in Figure 3.51 shows the interaction diagram of an application process with two subjects, an clerk and a head of department. Figure 3.52 shows the behavior diagrams of the clerk and head of department respectively. The positive or negative assessment of an application is transmitted as a message. The summary of reasons giving details of the assessment is only sent in the case of a negative assessment. If the application is confirmed, the "Summary of reasons" message is not transmitted, so we can assume that no further justification will be given in this case. 

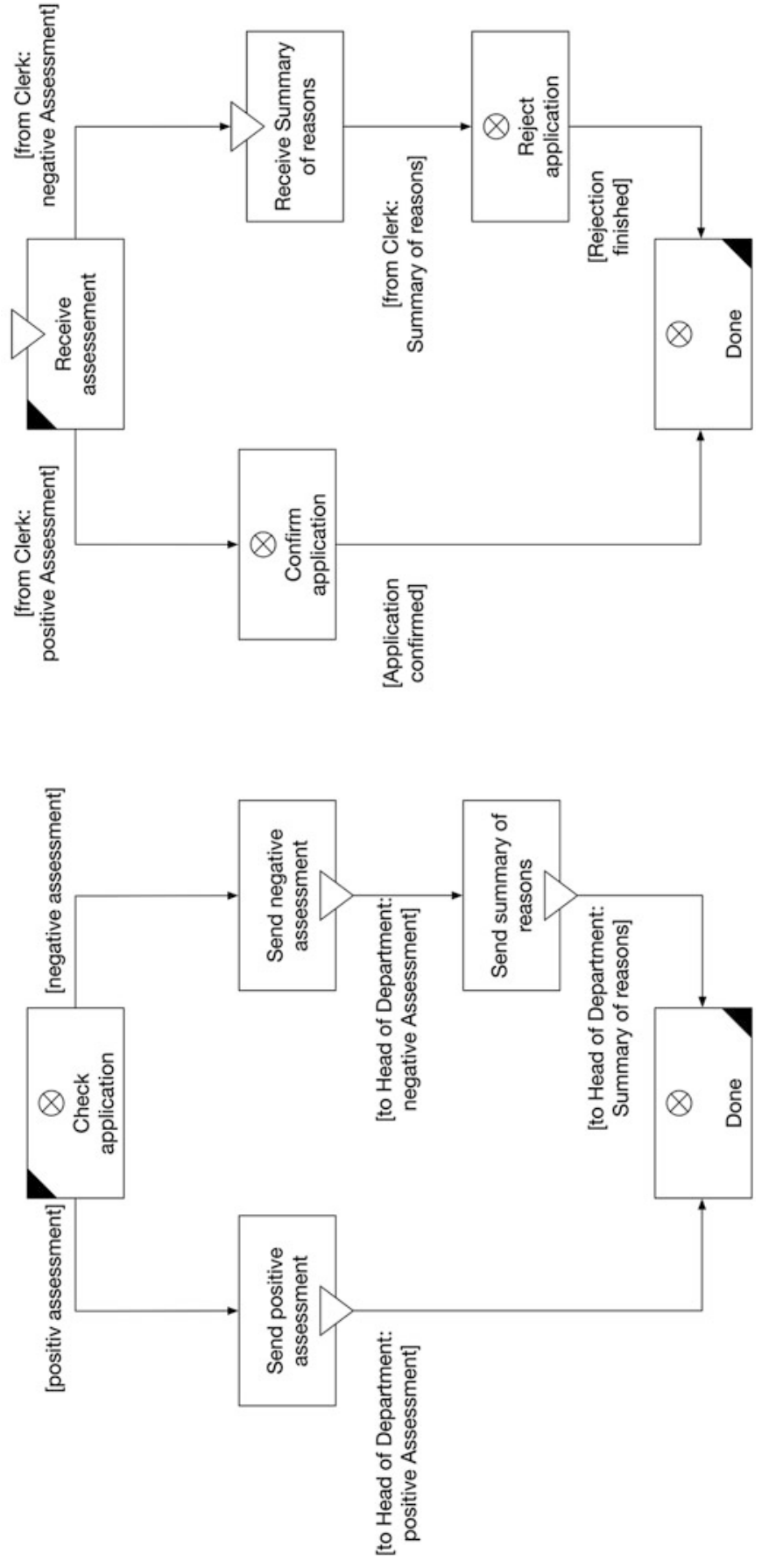

है 


\subsubsection{Advanced Forms of Communication Modeling and Exception Handling}

The focus of S-BPM on representing communication processes is reflected in a more comprehensive and flexible description of communication than in all the modeling languages considered above. In particular, S-BPM allows the representation of more complex communication scenarios through the use of input pools and the detailed description of the data exchanged in messages by means of business objects, as well as reacting on unforeseen messages by means of exception handling via message guards.

\section{Input pools}

An input pool provides a subject with a mailbox in which incoming messages are stored until they are required in the behavior diagram. In contrast to a simple mailbox, an input pool is configurable. For each subject, the number of storable messages can be specified per type. If the input pool is not able to accept a message according to its configuration, the sender must remain in the send state until the message can be delivered. This allows different communication scenarios to be represented. If the capacity of the input pool for a particular message type is reduced to 0 , the sender must always wait until the recipient is ready to receive the message. This is referred to as synchronous communication. If the input pool is configured to accept an arbitrary number of messages, the sender never has to wait until the receiver is in the state in which it can accept the message. This is called asynchronous communication (BPMN only allows the representation of this type of communication). Input pools also enable messages to be received in any order. The messages do not have to be processed in the order they arrive but can be processed according to the recipient's requirements.

Input pools have no graphical equivalent in S-BPM but are a concept of execution semantics. They are described for each subject textually or in a configuration tool. If no input pools are defined, the default configuration allows for an unlimited number of messages of any type to be stored. The communication behavior therefore corresponds to the message flows of BPMN (asynchronous communication).

\section{Business Objects}

Business objects are used to specify the data that is required for executing the tasks in a business process. Business objects are passive, i.e., they do not initiate any interactions or trigger any actions. Business objects are processed and modified by subjects and can be assigned to messages in order to specify their content in more detail.

As for input pools, there is no graphical equivalent for business objects in the notation of the modeling language. Business objects are concepts of execution semantics and therefore are dependent on the technical execution environment. They are usually described in tabular form. The basic structure of business objects consists of an identifier, data structures and data elements. The identifier of a 
business object is derived from the business environment in which it is used. Examples are business trip request, order, delivery note, invoice, etc.

Business objects consist of data structures whose components can be simple data elements of a certain type (for example, character string or number) or nested complex data structures. To allow for better comprehensibility, it is recommended to describe the meaning of the data elements in more detail, especially if the meaning cannot be derived from the identifiers.

Figure 3.53 shows an example of a business trip request. This consists, among other things, of the data structure 'Data on requester (employee)' with the data elements for last name, first name and personnel number, and the structure 'Data on business trip' with the data elements for start, end and purpose of the trip.

In many cases, the semantics of a business object can change during process execution, for example when a delivery note is transformed to an invoice. Several different statuses can therefore be defined for a business object. When the status is changed, only the data structures or data elements of the previous status that are

\begin{tabular}{|c|c|c|c|c|}
\hline Data structure & Meaning & Data type & Can/must & $\begin{array}{c}\text { Value } \\
\text { range/Default }\end{array}$ \\
\hline \multicolumn{5}{|l|}{ Data of requester } \\
\hline Name & Last name & Character & $M$ & \\
\hline First name & First name & Character & M & \\
\hline Personnel number & $\ldots$ & Integer & M & \\
\hline Organizational unit & $\ldots$ & & C & \\
\hline Pay group & $\ldots$ & & c & \\
\hline \multicolumn{5}{|l|}{ Data of trip } \\
\hline Start trip & $\cdots$ & Date & M & $\begin{array}{c}\text { Whitin } 1 \text { year from } \\
\text { current date/ } \\
\text { current date }\end{array}$ \\
\hline End trip & $\cdots$ & Date & M & $\begin{array}{l}\text { Start trip plus } 1 \\
\text { year/ start trip }\end{array}$ \\
\hline International trip & $\ldots$ & Boolean?? & C & $y / n ; n$ \\
\hline $\begin{array}{l}\text { Travel destination } \\
\text { (city7country) }\end{array}$ & $\ldots$ & Character & M & $\ldots$ \\
\hline Reason for traveling & $\ldots$ & Character & M & $\ldots$ \\
\hline $\begin{array}{l}\text { Desired advance } \\
\text { money }\end{array}$ & $\cdots$ & Integer & $\mathrm{C}$ & $\ldots$ \\
\hline \multicolumn{5}{|l|}{ Data of approval } \\
\hline Approval & Approval comment & Boolean?? & $M$ & $y / n ; n$ \\
\hline Cost center & $\ldots$ & Integer & M & $\ldots$ \\
\hline $\begin{array}{l}\text { Desired advance } \\
\text { money }\end{array}$ & $\cdots$ & Integer & C & $\cdots$ \\
\hline
\end{tabular}

Figure 3.53: Example of S-BPM business object (business trip request) 
required in the new status are transferred, and new components are added if required. This ensures that a subject only receives the data it really needs for its work. This also facilitates compliance with data protection regulations.

In the business trip request example, the status "Business trip booking" can be derived from the original status "Travel request" of the business object (cf., Figure 3.54). In particular, data elements with internal specifications such as personnel number, compensation group or reason for trip are removed, and thus do not to leave the company if the business object is sent to a travel agency for booking the travel arrangements. As shown in the following Figure 3.54, a new data structure "Booking data" is inserted for this purpose. It contains data elements with which a deadline can be specified for the travel agency to return booking confirmations or to specify certain hotel chains which are preferred by the company.

\section{Message Guards}

Handling of an exception (also termed message guard, message control, message monitoring, message observer) is a behavioral description of a subject that becomes relevant when a specific, exceptional situation occurs while executing a subject behavior specification. It is activated when a corresponding message is received, and the subject is in a state in which it is able to respond to the exception handling. In such a case, the transition to exception handling has the highest priority and will be enforced.

Exception handling is characterized by the fact that it can occur in a process in many behavior states of subjects. The receipt of certain messages, e.g., to abort the process, always results in the same processing pattern. This pattern would have to be modeled for each state in which it is relevant. Exception handlings cause high modeling effort and lead to complex process models, since from each affected state a corresponding transition has to be specified.

To illustrate the compact description of exception handlings, we use a service management process with the subject "service desk" (cf., Figure 3.55). This subject identifies a need for a business trip in the context of processing a customer order - an employee needs to visit the customer to provide a service locally. The subject "service desk" passes on a service order to an employee. Hence, the employee issues a business trip request. In principle, the service order may be canceled at any stage during processing up to its completion. Consequently, this also applies to the business trip application and its subsequent activities.

This relatively simple example already shows that taking such exception messages into account can quickly make behavior descriptions difficult to understand because additional receive states have to be added. In these additional receive states it is checked whether a corresponding cancel message has arrived. The concept of exception handling enables supplementing exceptions to the default behavior of subjects in a structured and compact form. Figure 3.55 below shows how such a concept affects the behavior of the employee.

Instead of incorporating additional receive states with a timeout zero in order to check whether a message has arrived which interrupts the standard control flow, the behavior description is enriched with an exception handling for the message "service 


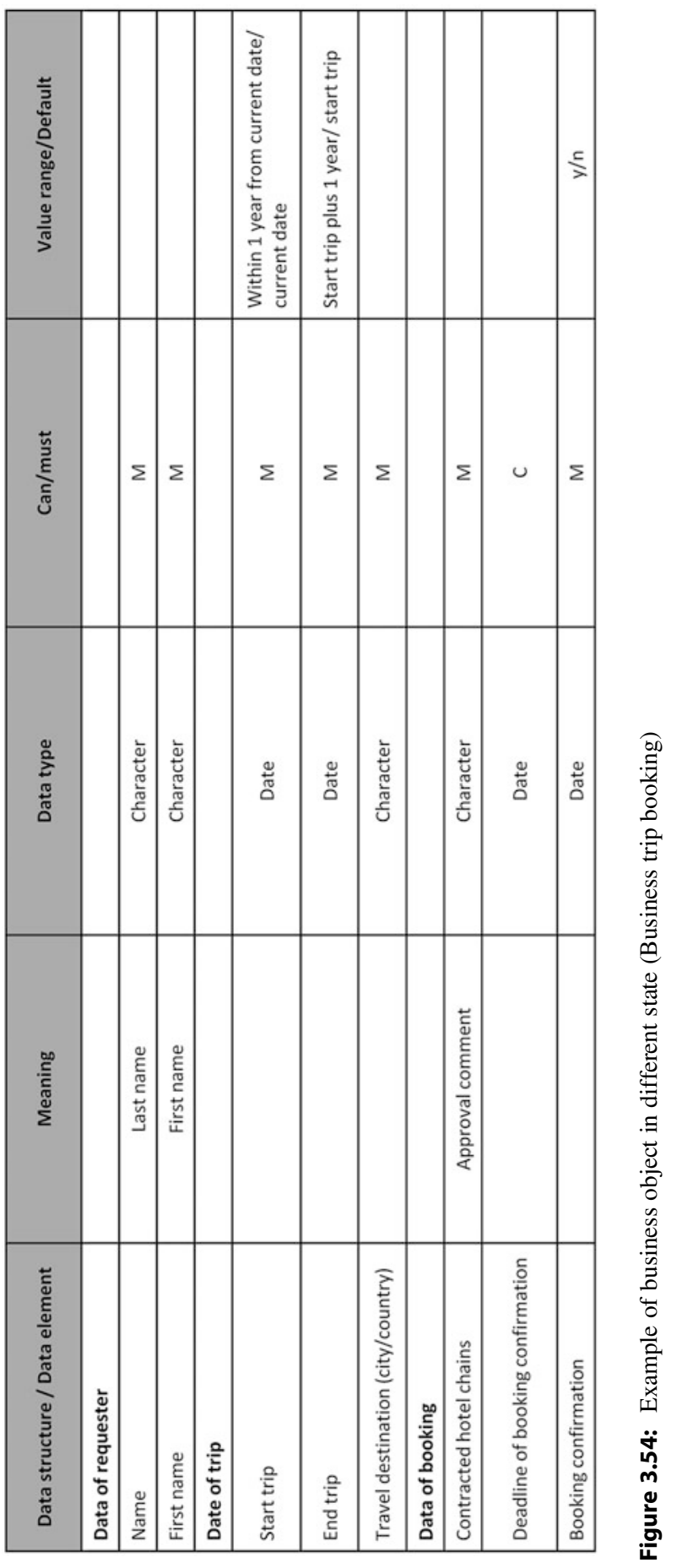




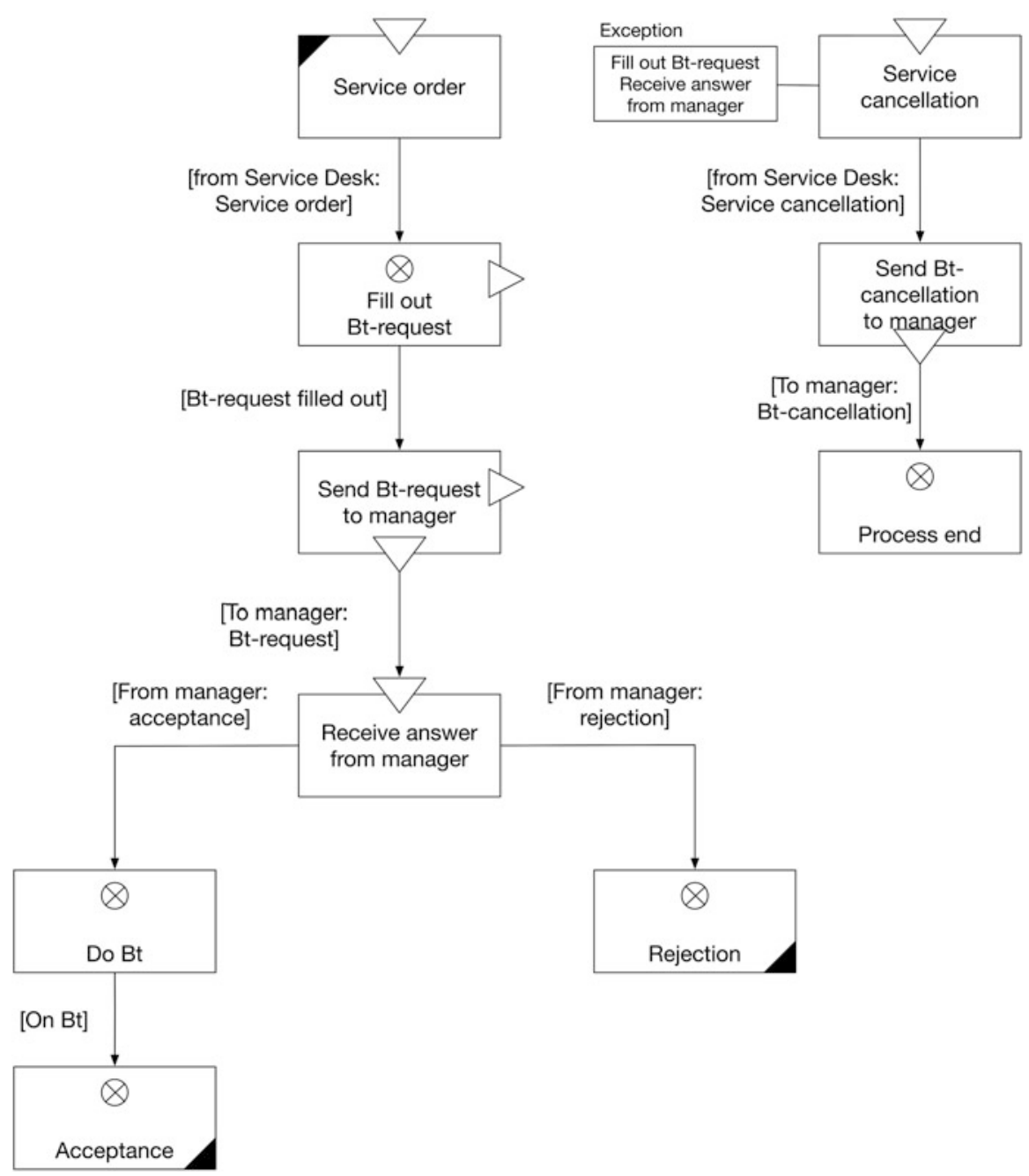

Figure 3.55: Behavior of subject "employee" with exception handling

cancellation". Its initial state is labeled with the states from which it is branched to, once the message 'service cancellation' is received. In the example, these are the states 'fill out Bt-request' and 'receive answer from manager'. Each of them is marked by a triangle on the right edge of the state symbol. The exception behavior leads to an exit of the subject, after the message 'service cancellation' has been sent to the subject 'manager'.

A subject behavior does not necessarily have to be brought to an end by an exception handling; it can also return from there to the specified default behavior. Exception handling behavior in a subject may vary, depending on from which state or by what type of message (cancellation, temporary stopping of the process, etc.) it 
is triggered. The initial state of exception handling can be a receive state or a function state.

Messages, like 'service cancellation', that trigger exception handling always have higher priority than other messages. This is how modelers express that specific messages are read in a preferred way. For instance, when the approval message from the manager is received in the input pool of the employee, and shortly thereafter the cancellation message, the latter is read first. This leads to the corresponding abort consequences.

Since now additional messages can be exchanged between subjects, it may be necessary to adjust the corresponding conditions for the input pool structure. In particular, the input pool conditions should allow storing an interrupt message in the input pool.

\section{Behavior Extensions}

When exceptions occur, currently running operations are interrupted. This can lead to inconsistencies in the processing of business objects. For example, the completion of the business trip form is interrupted once a cancellation message is received, and the business trip application is only partially completed. Such consequences are considered acceptable, due to the urgency of cancellation messages. In less urgent cases, the modeler would like to extend the behavior of subjects in a similar way, however, without causing inconsistencies. This can be achieved by using a notation analogous to exception handling. Instead of denoting the corresponding diagram with 'exception', it is labeled with 'extension'.

Behavior extensions enrich a subject's behavior with behavior sequences that can be reached from several states equivocally.

For example, the employee may be able to decide on his own that the business trip is no longer required and withdraw his trip request. Figure 3.56 shows that the employee is able to cancel a business trip request in the states 'send business trip request to manager' and 'receive answer from manager'. If the transition 'withdraw business trip request' is executed in the state 'send business trip request to manager', then the extension ' $\mathrm{F} 1$ ' is activated. It leads merely to canceling of the application. Since the manager has not yet received a request, he does not need to be informed.

In case the employee decides to withdraw the business trip request in the state 'receive answer from manager', then extension 'F2' is activated. Here, first the supervisor is informed, and then the business trip is canceled.

\section{Choice Segments}

So far, the behavior of subjects has been regarded as a distinct sequence of internal functions, send, and receive activities. In many cases, however, the sequence of internal execution is not important.

Certain sequences of actions can be executed in arbitrary order, this is called freedom of choice. In this case, the modeler does not specify a strict sequence of activities. Rather, a subject (or concrete entity assigned to a subject) will organize to a certain extent its own behavior at runtime. 


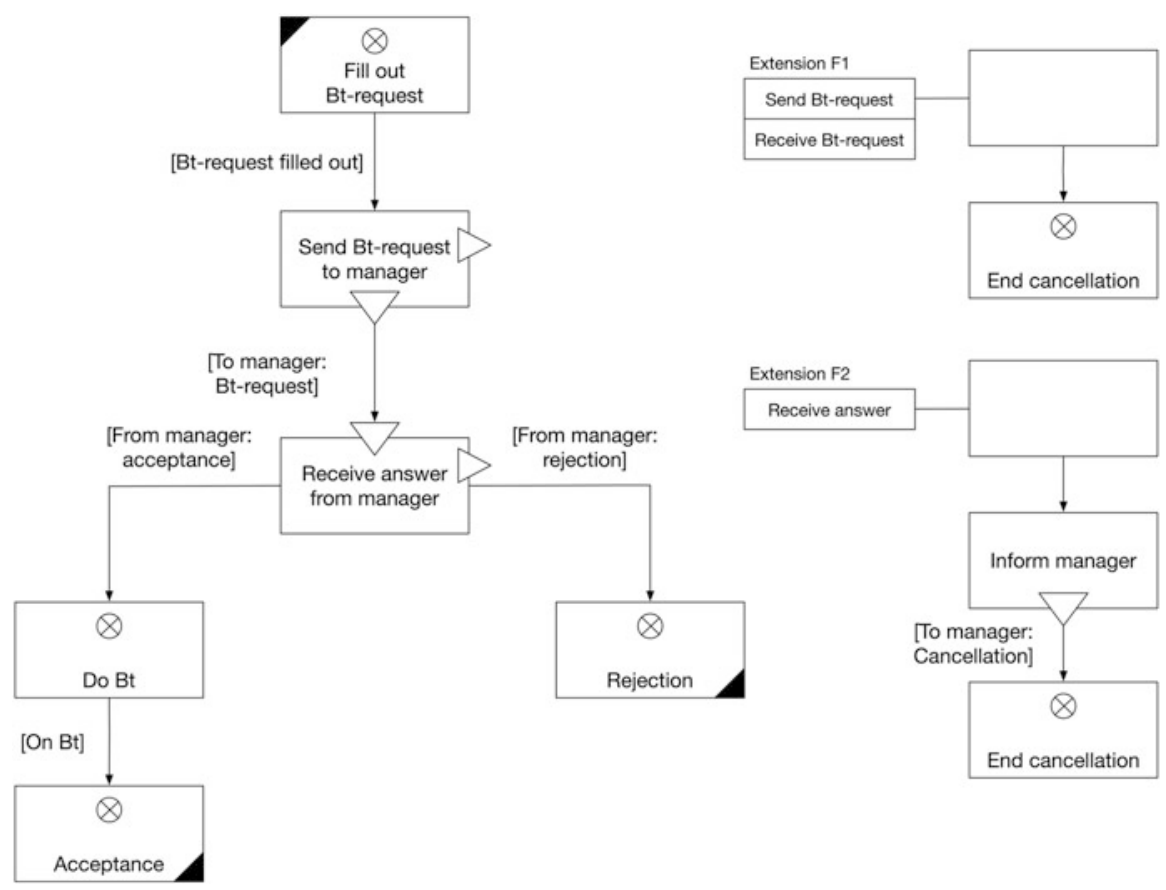

Figure 3.56: Example for S-BPM behavior extensions

The freedom of choice with respect to behavior is described as a set of alternative clauses which outline a number of parallel paths. At the beginning and end of each alternative, switches are used: A switch set at the beginning means that this alternative path is mandatory to get started, a switch set at the end means that this alternative path must be completely traversed. This leads to the following constellations:

- Beginning is set / end is set: Alternative needs to be processed to the end.

- Beginning is set / end is open: Alternative must be started, but does not need to be finished.

- Beginning is open / end is set: Alternative may be processed, but if so must be completed.

- Beginning is open / end is open: Alternative may be processed, but does not have to be completed.

The execution of an alternative clause is considered complete when all alternative sequences, which were begun and had to be completed, have actually been entirely processed and have reached the end operator of the alternative clause.

Transitions between the alternative paths of an alternative clause are not allowed. An alternate sequence starts in its start point and ends entirely within its end point. 


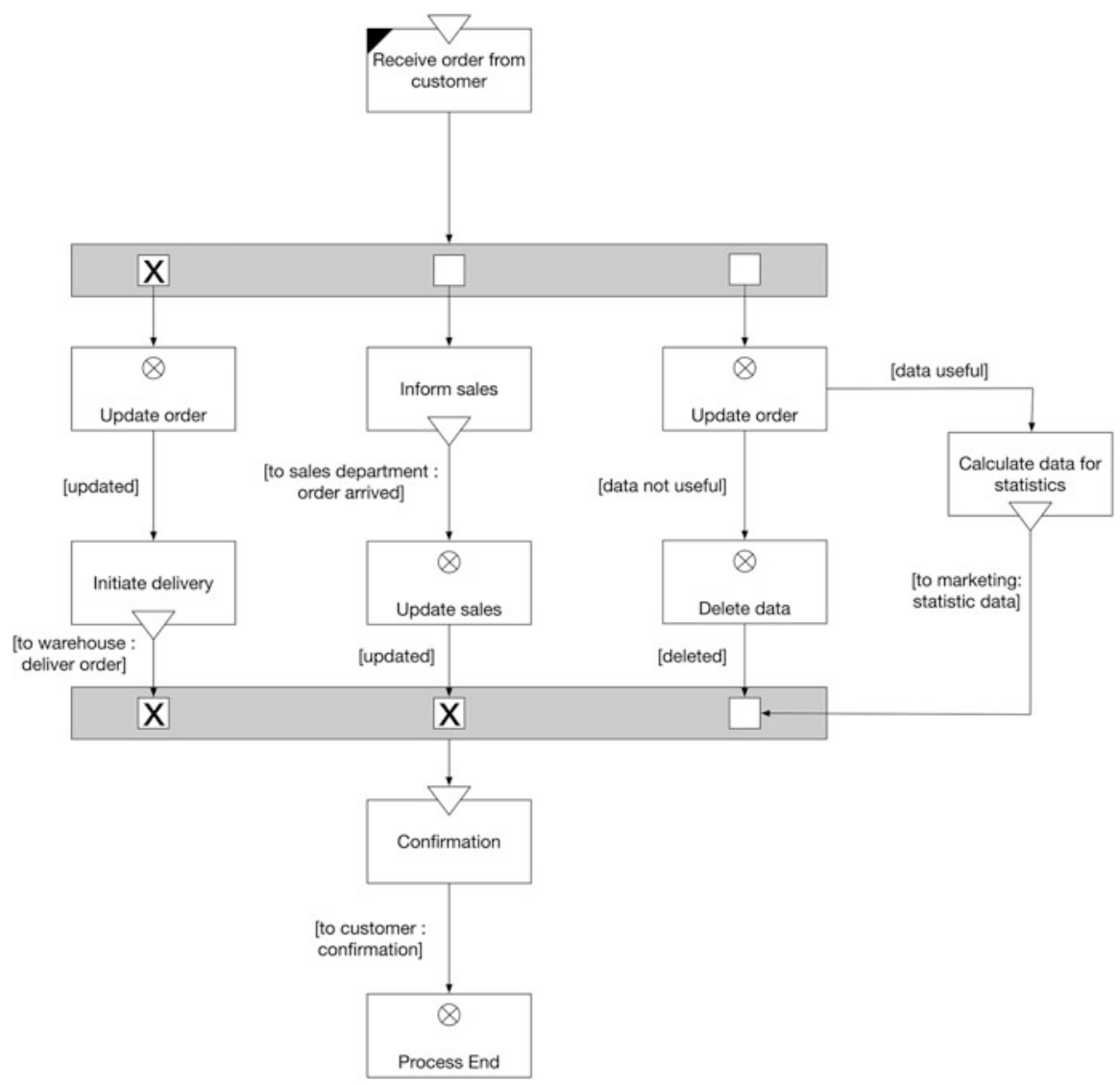

Figure 3.57: Example of process alternatives

Thus, the core property of a state chart, namely that a state chart always only contains a single active state is not violated

Figure 3.57 shows an example for modeling alternative clauses. After receiving an order from the customer, three alternative behavioral sequences can be started, whereby the leftmost sequence, with the internal function 'update order' and sending the message 'deliver order' to the subject 'warehouse', must be started in any case. This is determined by the ' $\mathrm{X}$ ' in the symbol for the start of the alternative sequences (gray bar is the starting point for alternatives). This sequence must be processed through to the end of the alternative because it is also marked in the end symbol of this alternative with an ' $\mathrm{X}$ ' (gray bar as the end point of the alternative).

The other two sequences may, but do not have to be, started. However, in case the middle sequence is started, i.e., the message 'order arrived' is sent to the sales department, it must be processed to the end. This is defined by an appropriate marking in the end symbol of the alternative (' $\mathrm{X}$ ' in the lower gray bar as the 
endpoint of the alternative). The rightmost path can be started but does not need to be completed. This kind of visualization radically simplifies the representation of the state transitions that would be necessary in case a traditional state chart visualization would be used (using a single connection for each potential state transition).

The individual actions in the alternative paths of an alternative clause may be arbitrarily executed in parallel and overlapping, or in other words: A step can be executed in an alternative sequence, and then be followed by an action in any other sequence. This gives the performer of a subject the appropriate freedom of choice while executing his actions.

\subsubsection{Classification}

In contrast to the other modeling languages discussed so far, there is no single diagram in S-BPM that fully describes a business process. Rather, separate behavior diagrams are created for all subjects, which are linked by an interaction diagram describing the message exchange. S-BPM thus enables a loose coupling of process parts and an easier adaptation of the behavior of a subject, as long as its communication interface, i.e., the set of received and sent messages and their sequence, remains unchanged.

The use of state diagrams to describe the behavior of a subject also constitutes a fundamental difference to the other languages discussed so far. A state diagram - as already indicated by its name - describes the state of a system (here: a subject - this can be a human as well as a machine) and the events that lead to a state transition. A subject can only be in exactly one state at any one time - it is therefore by definition not able to execute process steps in parallel. Rather, all subjects work in parallel and independently of each other. This requires a different approach to modeling, since constructs such as AND connectors (in EPCs), split/joins (in activity diagrams) or parallel gateways (in BPMN) are not available. At the same time, this modeling approach leads to simpler, more compact models and, in contrast to BPMN, a significantly reduced range of language constructs, which contributes to the comprehensibility of the models.

\subsection{Comparison}

The modeling languages considered here have different expressive power and, due to their historical development, have different focal points in their approach to represent business process [9]. The following section attempts to summarize these differences again systematically using the process definition presented in the former chapter and thus to compare the languages with respect to their expressiveness. We use the semantics of the presented modeling elements as a starting point.

The point of reference for the following considerations is the process definition from the last chapter, which we will reiterate here for simplicity's sake: 
Table 3.1: Concepts included in process definition

\begin{tabular}{l|l}
\hline Definition model & Concept \\
\hline $1 \mathrm{a}$ & Beginning \\
\hline & Input \\
\hline $\mathrm{b} \mathrm{b}$ & End \\
\hline $1 \mathrm{c}$ & Result \\
\hline $2 \mathrm{a}$ & Customers necessity \\
\hline $2 \mathrm{~b}$ & Activities / tasks \\
\hline & Start event \\
\hline $2 \mathrm{c}$ & Doer \\
\hline $2 \mathrm{~d}$ & Logical order \\
\hline $3 \mathrm{a}$ & Chronological order \\
\hline & Premise \\
\hline $3 \mathrm{~b}$ & Human \\
\hline & Machine \\
\hline & Material resources \\
\hline & Information \\
\hline & Application program \\
\hline & General aids \\
\hline
\end{tabular}

- Process strategy: A process has

- a defined start and input (start event),

- and has a defined end with a result,

- that contributes to the satisfaction of a customer's needs (and thus to the creation of value)

- Process logic: A process

- is the sum of linked activities (tasks),

- which, after the start event, are used by actors

- in logical and chronological order

- for processing a business object in order to

- generate the desired result.

- Process realization: A process is realized

- with people and/or machines, that take over the tasks of the respective actor, and carry these tasks out

- with tools (equipment, information, application programs, etc.).

On the basis of this definition, the concepts can be identified which should be representable in a process model in order to be able to model processes comprehensively (according to this definition). Table 3.1 shows these concepts. Concepts that occur more than once are only mentioned when they occur for the first time - such as "result". In the case of concepts which are described in different degrees of detail, only the more concrete concepts are considered - e.g., "linked activities" as a more general formulation of "logical and chronological order".

If one now assigns the modeling elements of the languages considered to these concepts, Table 3.2 results. 


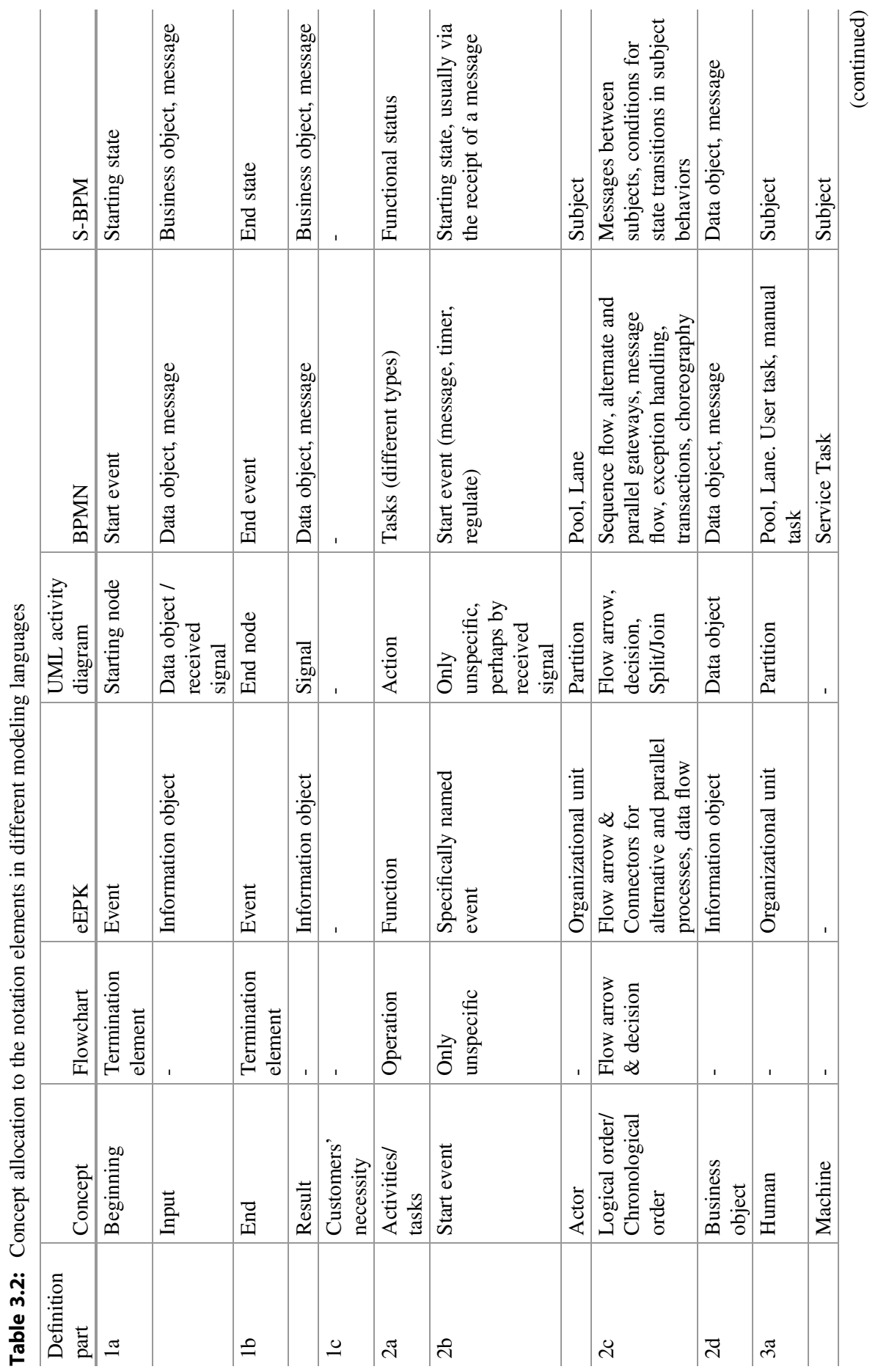




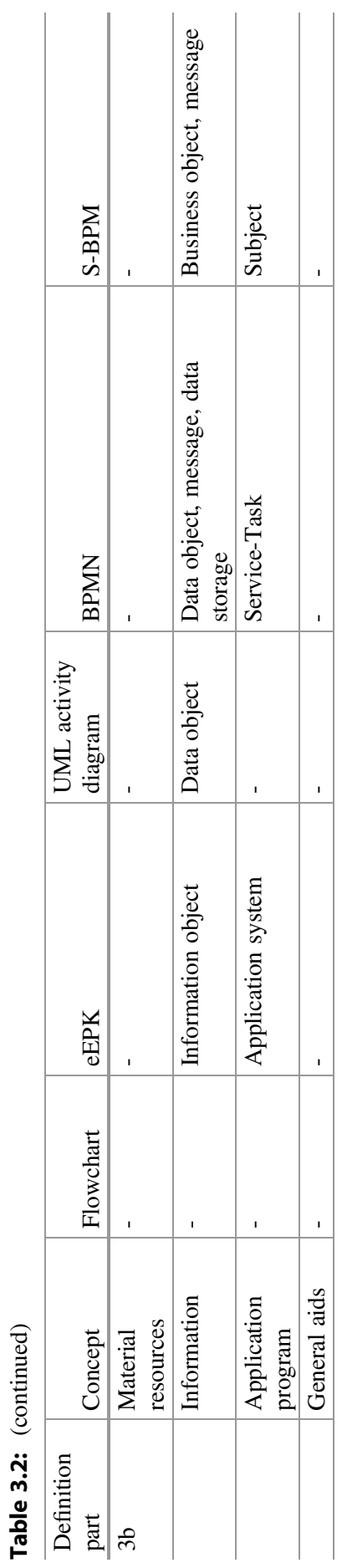


The conceptual coverage obviously varies across languages. The table also shows that not all languages address all concepts to the same extent or with the same level of expressiveness. The allocation of the modeling elements to the concepts provides a first starting point for estimating the expressiveness of the respective languages.

In this overview, the different approaches in the illustration of the logical and chronological connections are only partially recognizable. Here, the languages differ considerably: flowcharts do not offer the possibility of representing parallel processes, EPCs only allow for strong coupling of parallel activity branches by linking them within a process by means of AND or OR operators. UML activity diagrams and BPMN offer the same mechanisms (under different names), but also allow for loose coupling of processes or process parts by means of signals (for activity diagrams) or message flows (for BPMN). Especially the latter mechanism allows a detailed description of communication processes of basically independent process parts. Flexibility, however, is restricted by the necessary unique assignment of sender and receiver for each single message. S-BPM offers a similar communication mechanism, but is more flexible here (especially when using input pools that are not shown in the graphical representation of the language). In S-BPM, a description of process parts running in parallel is only possible by distributing them to different subjects - within a subject, only one functional state can be active at a time, i.e., only alternative branches in the behavior of a subject can be represented.

In general, BPMN offers the greatest flexibility in the choice of how to represent a process. Due to the large number of modeling elements, even complex real-world phenomena can be represented in a compact way. This, however, leads to higher demands on language comprehension for the model users. Activity diagrams or S-BPM, which are based on a compact set of modeling elements, follow a different approach here. Their approach leads to larger models in complex contexts, which in turn places higher demands on the model users with regard to their understanding of the model. S-BPM reduces the immediately visible complexity of models by distributing a process over different subjects. While this leads to partial models which can be more easily grasped, it in turn places higher demands on model users when it comes to grasping the overall context within the process.

When selecting a modeling language that is suitable for a given task and target group, not only the object of the model (i.e., the business process under consideration) and the target of modeling should be considered. The known or assumed competencies of the modelers and model users also need to be taken into consideration $[3,10,11]$. A fundamental distinction can be made between languages that focus on the flow of activity (such as flowcharts and EPCs) and those that focus on the actors in a process and their communication (such as S-BPM). BPMN and activity diagrams are basically suitable for both types of representation, whereby BPMN offers more expressive means for representing communication processes. The final selection of a modeling language after determining the fundamentally pursued representation approach (activity flow vs. communication flow) is ultimately dependent on the preferences of the modelers or model users. 


\section{References}

1. Curtis, B., Kellner, M. I., \& Over, J. (1992). Process modeling. Communications of the ACM, 35, 75-90. https://doi.org/10.1145/130994.130998.

2. Kunze, M., Luebbe, A., Weidlich, M., \& Weske, M. (2011). Towards understanding process modeling - The case of the BPM academic initiative. In International workshop on business process modeling notation (pp. 44-58). Berlin: Springer.

3. Recker, J., Safrudin, N., \& Rosemann, M. (2012). How novices design business processes. Information Systems, 37, 557-573. https://doi.org/10.1016/j.is.2011.07.001.

4. Herrmann, T., \& Loser, K. (1999). Vagueness in models of socio-technical systems. Behaviour \& Information Technology, 18, 313-323.

5. Nüttgens, M., \& Rump, F. (2002). Syntax und Semantik Ereignisgesteuerter Prozessketten (EPK). In Promise (pp. 64-77).

6. Dumas, M., \& ter Hofstede, A. (2001). UML activity diagrams as a workflow specification language. In UML 2001 - The unified modeling language modeling languages, concepts, and tools (pp. 76-90). Berlin: Springer.

7. White, S., \& Miers, D. (2008). BPMN modeling and reference guide: Understanding and using BPMN. The Journal of Strategic Information Systems, 3, 23-40. https://doi.org/10.1016/09638687(94)90004-3.

8. Fleischmann, A. (2010). What is S-BPM? In S-BPM ONE - Setting the stage for subjectoriented business process management (Vol. 85, pp. 85-106). Heidelberg: Springer.

9. Soderstrom, E., Andersson, B., Johannesson, P., et al. (2002). Towards a framework for comparing process modelling languages. In Proceedings of the 14th international conference on advanced information systems engineering (CAiSE) (Vol. 2348, pp. 600-611). Berlin: Springer. https://doi.org/10.1007/3-540-47961-9_41.

10. Oppl, S. (2018). Which concepts do inexperienced modelers use to model work? - An exploratory study. In Proceedings of MKWI 2018.

11. Soffer, P., Kaner, M., \& Wand, Y. (2012). Towards understanding the process of process modeling: Theoretical and empirical considerations. In Business process management workshops (pp. 357-369). Berlin: Springer.

Open Access This chapter is licensed under the terms of the Creative Commons Attribution 4.0 International License (http://creativecommons.org/licenses/by/4.0/), which permits use, sharing, adaptation, distribution and reproduction in any medium or format, as long as you give appropriate credit to the original author(s) and the source, provide a link to the Creative Commons licence and indicate if changes were made.

The images or other third party material in this chapter are included in the chapter's Creative Commons licence, unless indicated otherwise in a credit line to the material. If material is not included in the chapter's Creative Commons licence and your intended use is not permitted by statutory regulation or exceeds the permitted use, you will need to obtain permission directly from the copyright holder. 